\title{
Model-independent production of a top-philic resonance at the $\mathrm{LHC}$
}

\section{Nicolas Greiner, ${ }^{a, b}$ Kyoungchul Kong, ${ }^{c}$ Jong-Chul Park, ${ }^{c, d}$ Seong Chan Park ${ }^{d, e}$ and Jan-Christopher Winter ${ }^{b}$}

${ }^{a}$ DESY Theory Group, Notkestr. 85, Hamburg, D-22607 Germany

${ }^{b}$ Max Planck Institut für Physik, Föhringer Ring 6, München, D-80805 Germany

${ }^{c}$ Department of Physics and Astronomy, University of Kansas, Lawrence, KS, 66045 U.S.A.

${ }^{d}$ Department of Physics, Sungkyunkwan University, Suwon, 440-746 Korea

${ }^{e}$ School of Physics, Korea Institute for Advanced Study, Seoul, 130-722 Korea

E-mail: greiner@mpp.mpg.de, kckong@ku.edu, jcpark@ku.edu, s.park@skku.edu, jwinter@cern.ch

ABSTRACT: We investigate the collider phenomenology of a color-singlet vector resonance, which couples to the heaviest quarks, the top quarks, but very weakly to the rest of the fermions in the Standard Model. We find that the dominant production of such a resonance does not appear at the tree level - it rather occurs at the one-loop level in association with an extra jet. Signatures like $t \bar{t}$ plus jets readily emerge as a result of the subsequent decay of the resonance into a pair of top quarks. Without the additional jet, the resonance can still be produced off-shell, which gives a sizeable contribution at low masses. The lower top quark multiplicity of the loop induced resonance production facilitates its reconstruction as compared to the tree level production that gives rise to more exotic signatures involving three or even four top quarks in the final state. For all these cases, we discuss the constraints on the resonance production stemming from recent experimental measurements in the top quark sector. We find that the top-philic vector resonance remains largely unconstrained for the majority of the parameter space, although this will be scrutinized closely in the Run 2 phase of the LHC.

Keywords: Phenomenological Models, Hadronic Colliders

ARXIV EPRINT: 1410.6099 


\section{Contents}

1 Introduction 1

2 Bottom-up approach for a top-philic resonance $\left(G_{3}\right) \quad 3$

2.1 The setup 3

2.2 Tree level production 4

2.3 Loop induced production 9

2.3.1 Associated production: $p p \rightarrow G_{3}+j \rightarrow t \bar{t}+j \quad 9$

2.3.2 Off-shell production of the $G_{3}$ resonance: $p p \rightarrow G_{3} \rightarrow t \bar{t} \quad 11$

3 Current experimental bounds and future prospects for LHC Run $2 \quad 13$

$\begin{array}{ll}3.1 & \text { Bounds from searches for multiple top quark final states } \\ \end{array}$

$\begin{array}{ll}3.2 & \text { Bounds from uncertainties in top quark pair production measurements } \\ & 14\end{array}$

$\begin{array}{lll}3.2 .1 & \text { Inclusive jet cross sections } & 15\end{array}$

$\begin{array}{lll}3.2 .2 & \text { Exclusive jet cross sections and the } N_{\text {jets }} \text { distribution } & 17\end{array}$

$\begin{array}{lll}3.2 .3 & \text { Constraints on the loop level resonance production } & 18\end{array}$

$\begin{array}{lll}3.2 .4 & \text { LHC Run } 2 \text { prospects } & 23\end{array}$

4 Conclusions $\quad 24$

\section{Introduction}

With the recent discovery of a Higgs boson at the Large Hadron Collider (LHC) at CERN, we are beginning to explore the physics of the $\mathrm{TeV}$ scale in earnest. The next goal is the precise measurement of the properties of the newly discovered particle, and to find new physics beyond the Standard Model. The lightness of the discovered Higgs boson and precision measurements of Standard Model (SM) physics provide important guidance on building models beyond the SM. So far, the LHC has not seen any indication of new physics phenomena and the null results at the first run of the LHC provide stringent constraints on the mass scale of the new physics. Naively, one could imagine a scenario where the particle spectrum is relatively degenerate, or where new particles are simply out of reach for a proton-proton collider of $8 \mathrm{TeV}$ center-of-mass energy. However, it is also possible that new physics may be hiding in an exotic place and we have not been "digging" in the right spot yet. Therefore it is important to optimize searches based on some particular scenarios as well as to develop model-independent search strategies. Otherwise the new physics may be missed easily.

Among many others, searches for a new resonance (most likely originating from a new force) are particularly important. It may show up in an early stage of LHC Run 2 (with low luminosity) and it may be relatively easy to reconstruct the mass of the resonance. 
Typical final states, which are searched for so far, include dijet, dilepton, and diphoton topologies as well as signatures of $t \bar{t}$ pairs and so on. Current bounds on these resonances are already at scales of a few $\mathrm{TeV}$ for reasonable assumptions on couplings between SM particles and the resonance. Apart from the direct searches, indirect bounds on the scale of $t \bar{t}$ resonances have been discussed in the literature. In ref. [1] such bounds were derived from two-loop contributions to the oblique electroweak parameters $S$ and $T$. Although the new contributions may lead to constraints in the low mass region of the $G_{3}$, these indirect constraints turn out to be rather model-dependent. For this reason, we will not consider them in our study.

Especially searches for a $t \bar{t}$ resonance are very well motivated and many models contain this type of a resonance, such as the KK gluon [2,3] and KK graviton [4] in the RS model, the level-2 KK resonances in UED with bulk masses [5, 6], the coloron [7], the axigluon [8] and the $Z^{\prime}$ arising from a larger gauge symmetry. Current bounds on the $t \bar{t}$ resonance are quite strong and range from $2.0-2.5 \mathrm{TeV}$ for several models considered by the ATLAS and CMS collaborations [9-12]. Interestingly, in most experimental analyses, such a $t \bar{t}$ resonance is produced via an annihilation of $q \bar{q}$ pairs. However, if the $t \bar{t}$ resonance couples to the diquarks rather weakly or does not couple to them at all, one should consider that this resonance (which we label as " $G_{3}$ " in this paper) may be produced in association with another $t \bar{t}$ pair [13], i.e. $g g \rightarrow G_{3}+t \bar{t}$. This generates final states with four (decaying) top quarks, and in this case it would be challenging to reconstruct the resonance mass [13].

In this paper, we investigate the model-independent production of a top-philic resonance. We will assume that it dominantly couples to the top quark pair and rarely interacts with the rest of the SM particles, i.e. the diquark coupling is negligible. The resonance could be either a vector or a scalar; either a color-octet or a color-singlet. In this study, we will focus on a color-singlet vector particle.

We find that such a top-philic resonance can be produced in two different ways, that is at the tree level and at the one-loop level. The production via one loop leads to a large cross section compared to the tree level production modes. In the absence of the diquark coupling, the tree level production requires at least three particles in the final state, and is therefore suppressed by phase space. All these production modes are model-independent in a sense that they do not rely on how the resonance couples to other particles in the model. Even if a diquark coupling is zero, these production channels always exist as long as one is interested in a $t \bar{t}$ resonance.

A more exotic resonance, the chromophilic $Z^{\prime}$, is investigated in ref. [14] and has been searched for by the CDF collaboration [15]. In their study, this heavy $Z^{\prime}$ resonance interacts with the SM gluon only, leading to the dominant decay mode $Z^{\prime} \rightarrow g^{*} g \rightarrow q \bar{q} g$. In our study, we allow for an interaction of the $G_{3}$ to the top quark only and assume that there is no coupling to the SM gluon. Therefore, once produced, the $G_{3}$ vector particle will promptly decay into a pair of top quarks.

The outline of our paper is as follows: we set up a very simple model of a top-philic resonance and compute $G_{3}$ production cross sections in section 2 with emphasis on the production via one loop. In section 3 , we consider current bounds on the model parameter space and discuss the prospects of searching for $G_{3}$ in Run 2 of the LHC. Finally, we summarize our findings in section 4. 


\section{Bottom-up approach for a top-philic resonance $\left(G_{3}\right)$}

\subsection{The setup}

We consider a model with a color-singlet vector particle $\left(G_{3}\right)$ of mass $M_{G}$, which couples to the top quark ( $t$ and $\bar{t}$ ) only. We assume a very weak or no interaction with all the other quarks and the gluon. The only relevant interaction is given as follows:

$$
\begin{aligned}
\mathcal{L} & =\bar{t} \gamma_{\mu}\left(c_{L} P_{L}+c_{R} P_{R}\right) t G_{3}^{\mu}, \\
& =c_{t} \bar{t} \gamma_{\mu}\left(\cos \theta P_{L}+\sin \theta P_{R}\right) t G_{3}^{\mu},
\end{aligned}
$$

where $P_{L / R}=\left(1 \mp \gamma_{5}\right) / 2, c_{t}=\sqrt{\left(c_{L}\right)^{2}+\left(c_{R}\right)^{2}}$ and $\tan \theta=\frac{c_{R}}{c_{L}}$. The decay width at leading order is given by

$$
\begin{aligned}
\Gamma\left(G_{3} \rightarrow t \bar{t}\right) & =\frac{c_{t}^{2} M_{G}}{8 \pi} \sqrt{1-\frac{4 m_{t}^{2}}{M_{G}^{2}}}\left[1+\frac{m_{t}^{2}}{M_{G}^{2}}(3 \sin 2 \theta-1)\right], \\
& \approx \frac{c_{t}^{2} M_{G}}{8 \pi} \quad \text { for } m_{t} \ll M_{G} .
\end{aligned}
$$

Since $\frac{\Gamma}{M_{G}} \approx \frac{c_{t}^{2}}{8 \pi} \ll 1$, the width of the resonance is very narrow for $c_{t} \sim 1$, and therefore determined by the detector resolution. Throughout this paper, we will only consider the two-body decay mode $G_{3} \rightarrow t \bar{t}$ where $M_{G}>2 m_{t}$. Note that in principle the $G_{3}$ can decay into a $t W \bar{b}$ system or into a $W^{+} b W^{-} \bar{b}$ system if $m_{t}+M_{W}+m_{b}<M_{G}<2 m_{t}$ or $2 M_{W}+2 m_{b}<M_{G}<m_{t}+M_{W}+m_{b}$, respectively. This is shown in figure 1. The solid curve (in blue) has been computed considering the four-body final state $G_{3} \rightarrow W^{+} W^{-} b \bar{b}$, while the $t \bar{t}$ final state with on-shell top quarks is shown as (red) circles. Beyond the $t \bar{t}$ threshold, both calculations agree.

In our setup, there are only three parameters, the mass of the $t \bar{t}$ resonance $\left(M_{G}\right)$ and the interaction strengths, i.e. $\left(c_{L}, c_{R}\right)$ or $\left(c_{t}, \theta\right)$. We will take a bottom-up approach using the above Lagrangian without considering any underlying theory. Therefore we will not assume any theoretical constraints in our parameters and will only consider experimental bounds. However, each underlying model may provide different constraints and certain parameter regions may be prohibited. For instance, a KK gluon in the RS model tends to be very heavy and its couplings are determined by the wave function overlap in extra dimensions.

For our model-independent approach, we find that a top-philic resonance as advocated here can be produced in two different ways:

1. at the tree level where we have three main production channels for a $G_{3}$ resonance:

(a) the $G_{3} t \bar{t}$ channel yielding a four top-quark final state, $p p \rightarrow G_{3}+t \bar{t} \rightarrow t \bar{t}+t \bar{t}$,

(b) the $G_{3} t j$ channel yielding a three top-quark final state, $p p \rightarrow G_{3}+t / \bar{t}+j \rightarrow$ $t \bar{t}+t / \bar{t}+j$,

(c) and the $G_{3} t W$ channel yielding, again, a three top-quark final state, $p p \rightarrow$ $G_{3}+t / \bar{t}+W^{ \pm} \rightarrow t \bar{t}+t / \bar{t}+W^{ \pm}$ 


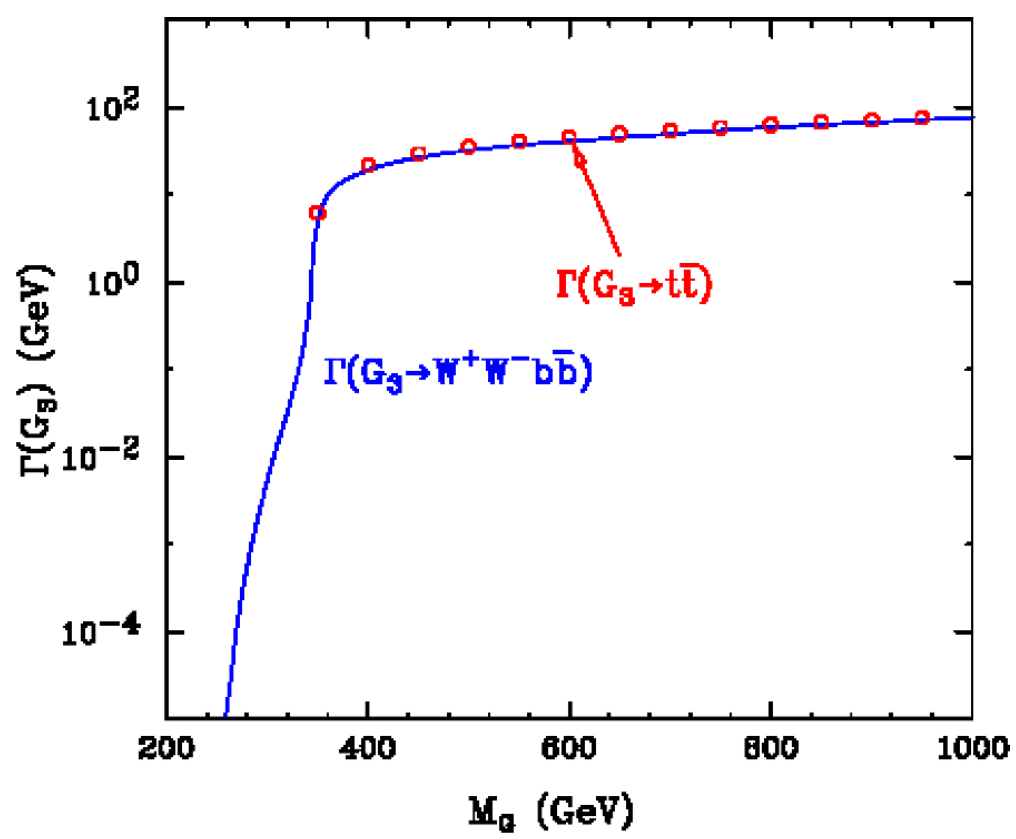

Figure 1. Total decay width of $G_{3}$. The solid curve (in blue) represents the results of the computation of the four-body decay $G_{3} \rightarrow W^{+} W^{-} b \bar{b}$, while the two-body decay yielding two on-shell top quarks is shown by the circles (in red).

2. at the one-loop level where we identify two production modes leading to di-top-quark final states, namely:

(a) the loop induced $G_{3} j$ production, $p p \rightarrow G_{3}+j \rightarrow t \bar{t}+j$,

(b) and the loop induced $G_{3} \rightarrow t \bar{t}$ production from an off-shell $G_{3}, p p \rightarrow G_{3} \rightarrow t \bar{t}$ (off-shell).

We will discuss all of these production channels in more detail below.

\subsection{Tree level production}

In this section, we consider the tree level production of the $G_{3}$ resonance, which always involves at least one extra (i.e. non-decay) top quark in the final state. The basic structure of this production stems from top quark production in the SM, see figure 2, where the top quark may be produced singly $\left(t j / \bar{t} j\right.$ and $\left.t W^{-} / \bar{t} W^{+}\right)$or in a pair $(t \bar{t}) .{ }^{1}$ Subsequently, these top quarks in the final state radiate a $G_{3}$, which will decay further into a $t \bar{t}$ pair. In the case of $t \bar{t}$ and $t W$ production, there are additional contributions from $\hat{t}$-channel radiation as shown by the left and right example diagrams of figure 2, respectively.

Figure 3 summarizes the tree level production cross sections of the $G_{3}$ as a function of its mass $\left(M_{G}\right)$ for a choice based on natural coupling values, $c_{L}=1=c_{R}$. We use CALCHEP [16] for the cross section evaluation and verified the results using MADGraph5 [17]. We set the renormalization and factorization scale to $\mu_{0}=\sqrt{\hat{s}} / 2$, and

\footnotetext{
${ }^{1}$ Our definition of a jet $(j)$ contains the gluon and all five quarks $(q)$ as well as their anti-particles. Note that the gluon $(g)$ does not appear as a final state parton in the tree level production shown here.
} 


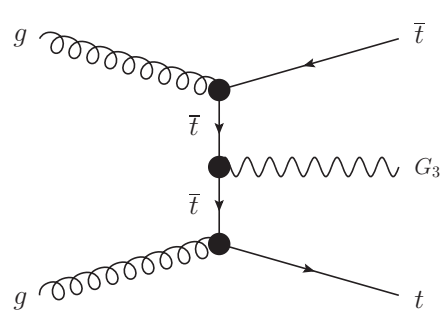

(a)

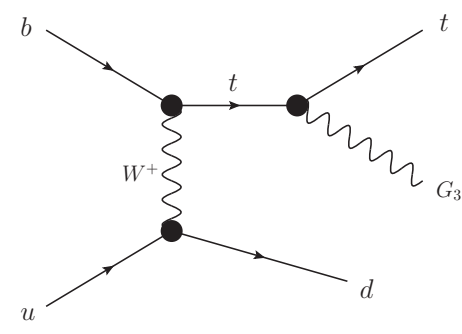

(b)

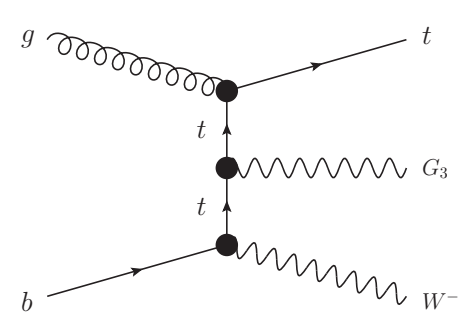

(c)

Figure 2. Sample diagrams for the $G_{3}$ tree level production in association with (a) $t \bar{t}$, (b) $t j$ and (c) $t W$. The $G_{3}$ generation modes are derived from top quark final states produced via strong (a), electroweak (b) and mixed QCD and electroweak (c) interactions.

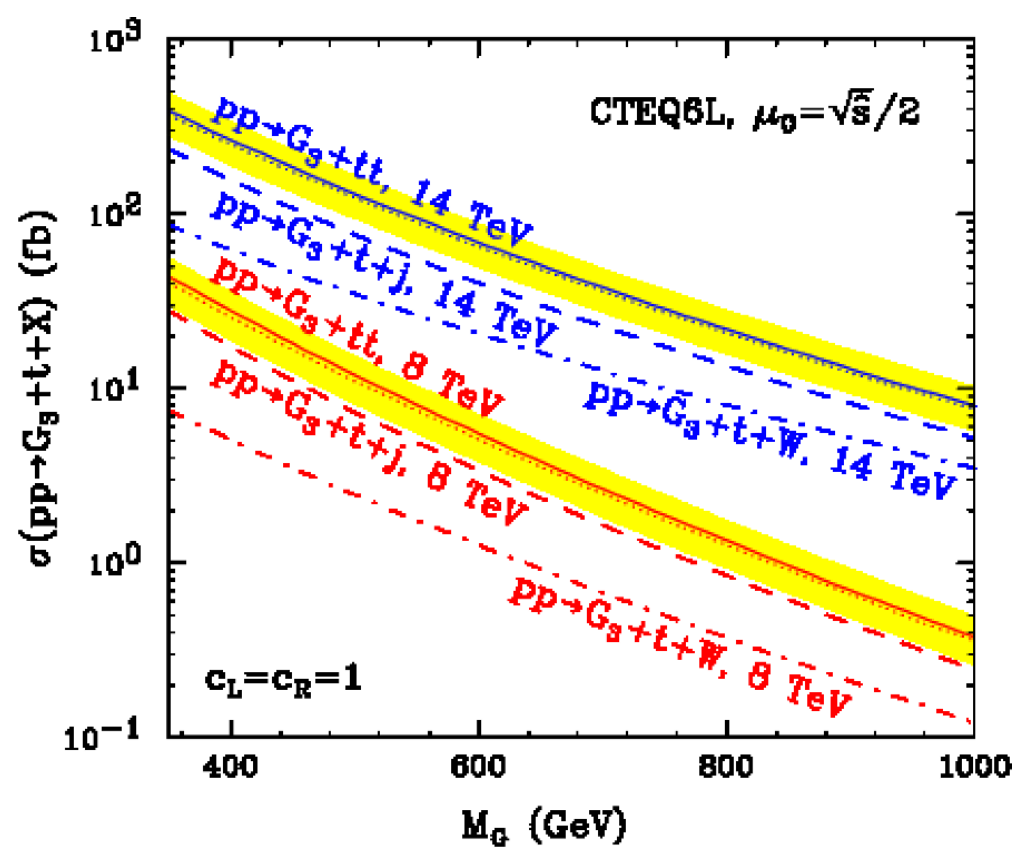

Figure 3. Tree level production cross sections of the $G_{3}$ vector particle in association with $t \bar{t}$ (solid), $t j$ (dashed) and $t W$ (dot-dashed) at the LHC, using natural values for the coupling strengths $\left(c_{L}=c_{R}=1\right)$. The cross sections are shown as a function of the mass of the resonance, $M_{G}$; the yellow bands correspond to scale variations by factors of two.

employ the CTEQ6L parton density functions for all computations. Especially for the $G_{3} t \bar{t}$ production, this scale choice gives consistent results compared to the one reading $\mu_{0}=M_{G} / 2+m_{t}$, which is similar to $M_{h} / 2+m_{t}$ used in $t \bar{t} h$ production. The cross section results for the processes $p p \rightarrow G_{3}+t \bar{t}, p p \rightarrow G_{3}+t j$ and $p p \rightarrow G_{3}+t W$ are shown as solid, dashed, and dot-dashed curves, respectively, with the $8 \mathrm{TeV}$ results depicted in blue and the $14 \mathrm{TeV}$ results depicted in red. No cuts have been imposed in figure 3 and all cross sections are compared at the parton level.

For $p p \rightarrow G_{3}+t \bar{t}$ (solid lines in figure 3), there are 112 diagrams (including electroweak processes such as $\left.q \bar{q} \rightarrow Z \rightarrow t \bar{t}+G_{3}\right)$. Among these there are 8 diagrams associated with 
the $g g \rightarrow G_{3}+t \bar{t}$ subchannel, which turns out to give the dominant contribution as shown by the dotted curves in figure 3. The gluon initial state contribution accounts for about $94 \%(90-95 \%)$ of the total production cross section at $14 \mathrm{TeV}(8 \mathrm{TeV})$. The yellow band represents the uncertainty that arises by varying the scales between $\mu_{0} / 2$ and $2 \mu_{0}$. Four top-quark final states arise in many models beyond the SM. Examples include the top compositeness model [18, 19], the topcolor-assisted technicolor model [20], and models that produce a pair of $t \bar{t}$ resonances such as the axigluon, the KK gluon [21], the coloroctet scalar [22, 23] and the color-sextet scalar [24]. The production of four top-quark final states, which we propose here, is similar to that of $t \bar{t} H$, where $H$ is a heavy neutral Higgs boson, which decays to a top quark pair.

The electroweak production of a $G_{3}+t j$ (dashed lines in figure 3 ) is mediated by a $W$ boson as exemplified in the middle diagram in figure 2 . The major part of the cross section comes from the initial states with a $b$-quark, which can be understood in terms of the PDF and CKM structure. Similarly, the $g$ plus $b$-quark initial states provide the dominant contribution to the $G_{3} t W$ production (dot-dashed lines in figure 3 ).

The $G_{3} t W$ production is the smallest owing to the suppression from the three-body phase space and the heaviness of the final state particles. $G_{3} t j$ is slightly larger than $G_{3} t W$ due to the jet multiplicity and the lightness of jets compared to the $W$ boson. The $G_{3} t \bar{t}$ channel gives the largest cross section due to the fact that the top quark pair production is mainly governed by the strong interaction, overpowering the effect from the phase-space suppression.

We find that the $G_{3}$ production associated with a single top quark exhibits a strong dependence on the chirality as shown in figures 4 and 5 , while the $G_{3}$ production accompanied by a top quark pair does not change as a function of chirality (see figure 6). The difference between these two findings stems from the fact that $G_{3} t W$ and $G_{3} t j$ production is mediated by the $t-W-b$ interaction. For these processes, $\theta=0(\theta=\pi / 2)$ corresponds to the pure left-handed (right-handed) interaction and leads to the largest (smallest) cross section for a given mass of the $G_{3}$. On the contrary, the $G_{3} t \bar{t}$ process does not possess a $t-W-b$ coupling and we checked analytically that in this case the dependence on the chirality angle $\theta$ drops out. Note that the cross sections (based on $c_{t}=1$ ) in figures $4-6$ are consistent with the results shown in figure 3, taking the change from $c_{t}=1 \rightarrow c_{t}=\sqrt{2}$ into account.

In the literature, only $G_{3} t \bar{t}$ production is studied owing to its large cross section but we stress that the cross section for $G_{3} t j$ is smaller only by a factor of two and it provides a good opportunity at the $14 \mathrm{TeV}$ LHC. Especially for resonance structure searches in an inclusive channel such as in multi-lepton final states, all production modes contribute at a non-negligible rate. The inclusive search is important as the tree level production leads to multi-gauge boson (or multi-top quark) final states, which make it difficult to reconstruct a bump in a straightforward manner. Figure 7 shows the number of expected signal events in the inclusive dilepton, same-sign dilepton and trilepton final state. The cross sections have been computed for $c_{t}=1$ and are normalized to $1 \mathrm{fb}^{-1}$ at $14 \mathrm{TeV}$. The four-lepton events are roughly $5-6 \%$ of the events that have at least three leptons in the final state. As shown in figure 7 , the same-sign dilepton events are fairly large and make up a fraction of about 


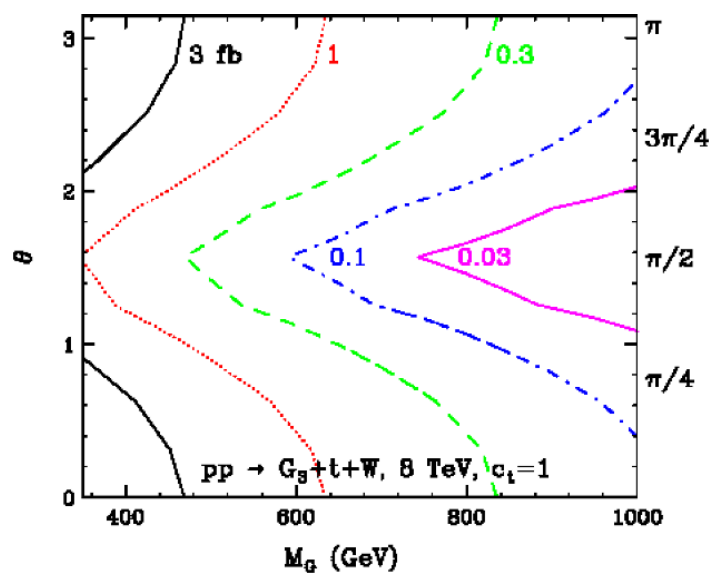

(a)

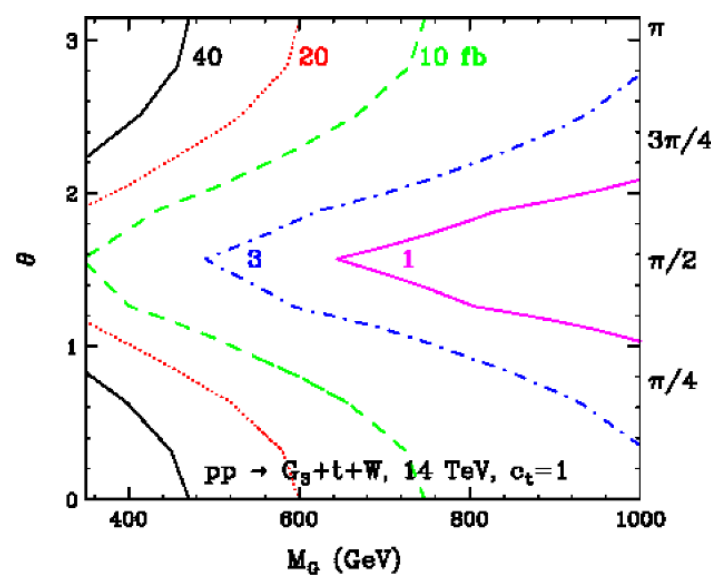

(b)

Figure 4. Tree level production cross section of $p p \rightarrow G_{3}+t W$ at $8 \mathrm{TeV}$ (4a) and at $14 \mathrm{TeV}$ (4b) in the $M_{G}-\theta$ plane of model parameters using $c_{t}=1$.

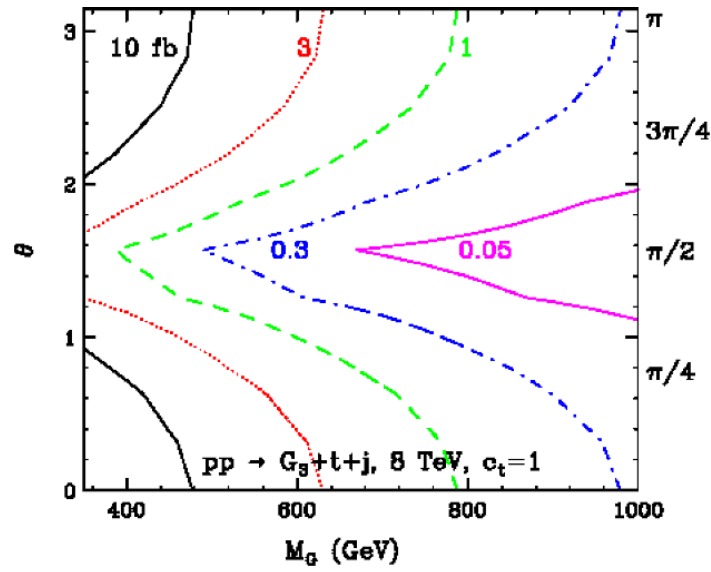

(a)

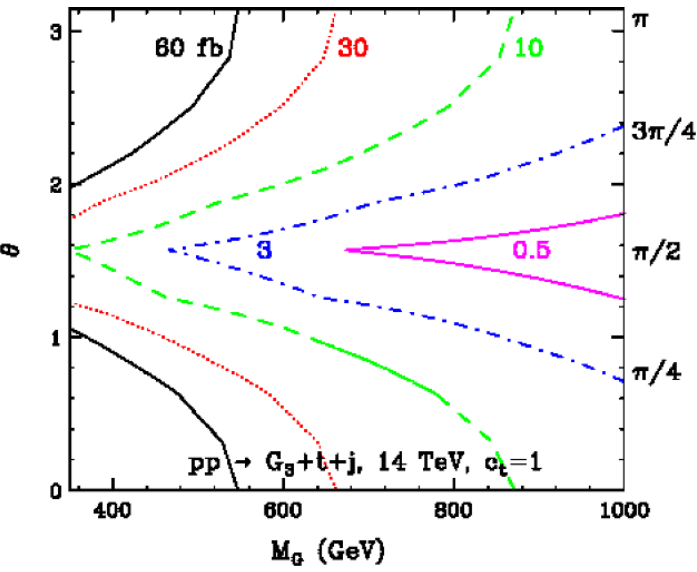

(b)

Figure 5. Tree level production cross section of $p p \rightarrow G_{3}+t j$ at $8 \mathrm{TeV}(5 \mathrm{a})$ and at $14 \mathrm{TeV}(5 \mathrm{~b})$ in the $M_{G}-\theta$ plane of model parameters using $c_{t}=1$.

$40-45 \%$ of the inclusive dilepton events. The statistics for dilepton events is higher than for trilepton events by a factor of 7 but a larger background is expected for the dilepton selection. Note that all curves in figure 7 are just rough estimates of such event counts, since no cuts have been imposed in this study.

We finally remark that the above discussion of the tree level generation of the $G_{3}$ resonance has been guided by the principle of modifying resonant SM top quark topologies in single top quark (electroweak interaction) and top quark pair (strong interaction) production by permitting the real emission of a $G_{3}$ particle off one of the top quark lines. However, as one implication of a more complete physics picture we immediately realize that the $G_{3}$ can also emerge from SM topologies with non-resonant top quarks. For example, in the $g g \rightarrow b W^{+} W^{-} \bar{b}$ process, the $G_{3}$ can be attached to a $\hat{t}$-channel top quark propagator 


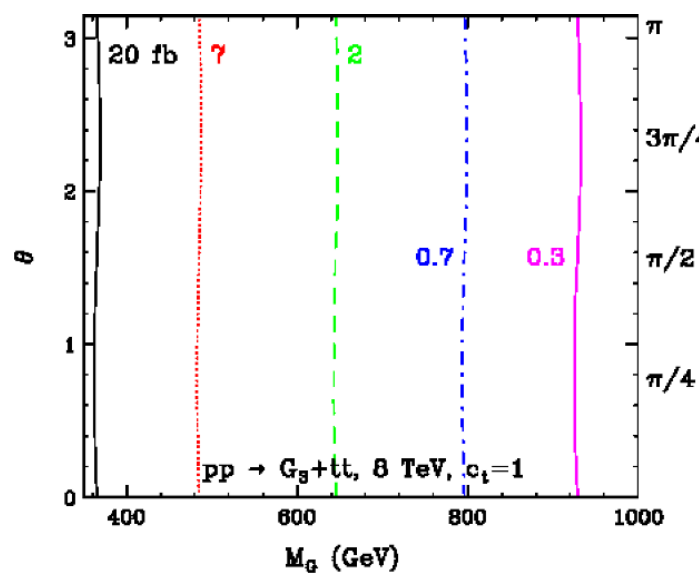

(a)

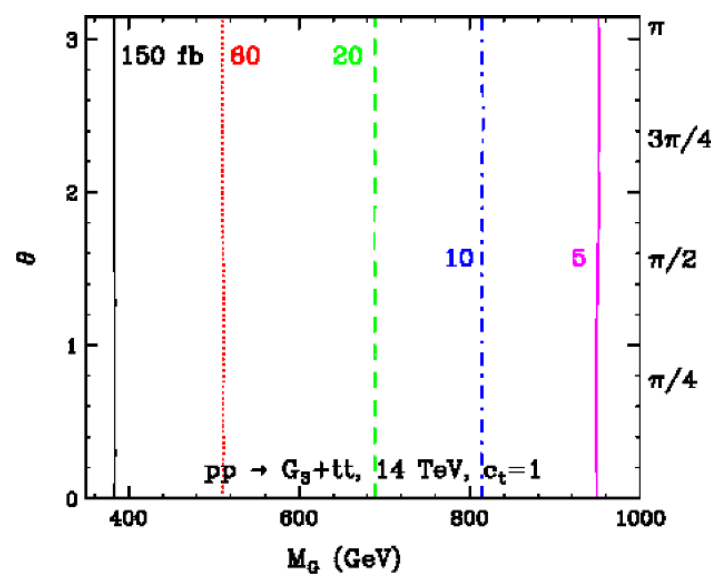

(b)

Figure 6. Tree level production cross section of $p p \rightarrow G_{3}+t \bar{t}$ at $8 \mathrm{TeV}(6 \mathrm{a})$ and at $14 \mathrm{TeV}(6 \mathrm{~b})$ in the $M_{G}-\theta$ plane of model parameters using $c_{t}=1$.
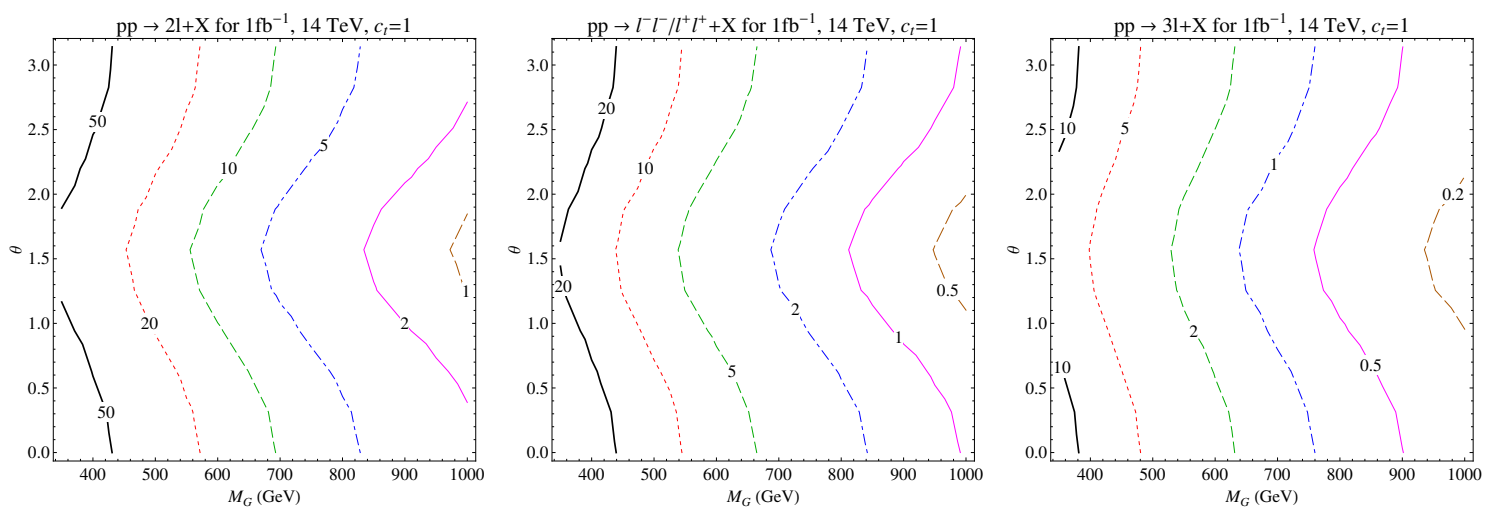

Figure 7. The expected number of events from decays of tree level generated $G_{3}$ resonances that contribute to final states for the inclusive production of dileptons (left), same-sign dileptons (middle), and trileptons (right).

connecting two ingoing $b$-quarks each of which originating from an initial state gluon splitting and emitting a $W$ boson subsequently. At the inclusive level — which we discuss in this paper - non-resonant contributions like the one in this example are usually small and can be neglected. However, one should bear in mind that more sophisticated kinematic selections project out phase-space regions where these contributions may become relatively large. Furthermore, a $G_{3}$ emission can shift the top quark propagator onto its mass-shell. In these cases, it is important to go beyond the approximation of factorizing the top quark and $G_{3}$ production from their decays. For instance, the $G_{3} t W$ and $G_{3} t \bar{t}$ are the singly and doubly top quark resonant parts of just one, more inclusive, more physical, $G_{3} W^{+} b W^{-} \bar{b}$ calculation (in the four-flavour scheme). This is very similar to realizing that the $t W$ and $t \bar{t}$ configurations are contained in the calculation of the one SM process $p p \rightarrow W W b \bar{b}$ in the four-flavour scheme. 


\subsection{Loop induced production}

In this section, we focus on the new class of production modes, which, as mentioned before, only arise at the one-loop level.

The virtual amplitudes at one loop have been generated with GoSAm [25, 26], a publicly available package for the automated generation of one-loop amplitudes. It is based on a Feynman diagrammatic approach using QGRAF [27] and FORM [28] for the diagram generation, and SpINney [29], Haggies [30] and FORM to write an optimized Fortran output. For the reduction of the tensor integrals, we used NINJA [31-33], an automated package for the integrand reduction via Laurent expansion. This package is a part of GoSAm and therefore no further work is required to use it. Alternatively, one can use other reduction techniques such as integrand reduction using the OPP method [34-36] as implemented in SAMURAI [37] or methods of tensor reduction as contained in GolEM95 [3841]. The remaining scalar integrals have been evaluated using OnELoOP [42].

We can obtain the diagrams involving a $G_{3}$ resonance by using a SM $Z$ boson, require that the $Z$ boson couples only to top quarks, and modify its parameters such as the mass, the width, and the couplings to vector- and axial-currents accordingly. All diagrams for all subprocesses and production modes are ultraviolet and infrared finite, i.e. all possible double and single poles of the one-loop amplitude are zero. In GoSAm this "zero" is obtained numerically which means deviations from zero can be used to assess possible numerical instabilities. Therefore we checked the pole terms for each phase-space point and any event was rejected if the pole terms were in agreement with zero in less than thirteen digits. The fraction of such events was however in the sub per-mill range which indicates a numerically stable evaluation of the amplitudes.

For the numerical integration over the phase space, we used MadEvent [43]. To improve on the timing for the evaluation of a phase-space point, we introduced a Monte Carlo sampling over the helicities for the gluon initiated subprocess in the $t \bar{t}+$ jet channel (see section 2.3.1).

For the theoretical predictions, we used the following setup and parameters:

$$
p_{T, j}>25 \mathrm{GeV}, \quad\left|\eta_{j}\right|<2.5, \quad R=0.4, \quad m_{t}=173.4 \mathrm{GeV}, \quad \Gamma_{t}=1.5 \mathrm{GeV}
$$

Both the renormalization and factorization scale were set to $\mu_{\mathrm{R}, \mathrm{F}}=\sqrt{\hat{s}} / 2$, and we used the CTEQ6L PDF set. Concerning the jet definition, this setup defines in particular what we will refer to as the experimental cuts. Later on, we will also make use of the loose cuts regime where we relax the requirements on the jets reading

$$
p_{T, j}>20 \mathrm{GeV} \quad \text { and } \quad\left|\eta_{j}\right|<6.0 .
$$

\subsubsection{Associated production: $p p \rightarrow G_{3}+j \rightarrow t \bar{t}+j$}

Two different subprocesses contribute, namely

$$
\begin{aligned}
& g g \rightarrow G_{3}(\rightarrow t \bar{t})+g, \\
& q \bar{q} \rightarrow G_{3}(\rightarrow t \bar{t})+g,
\end{aligned}
$$




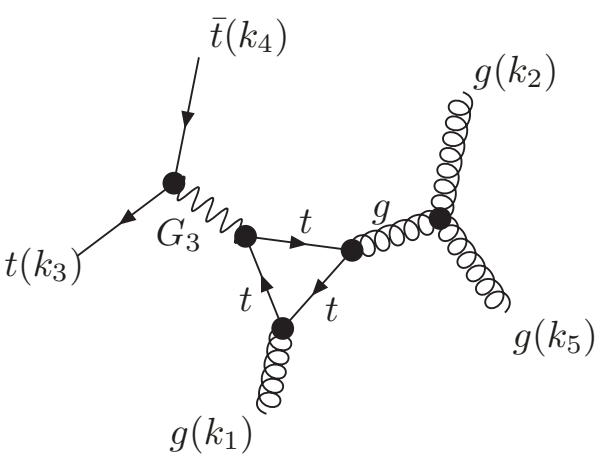

(a)

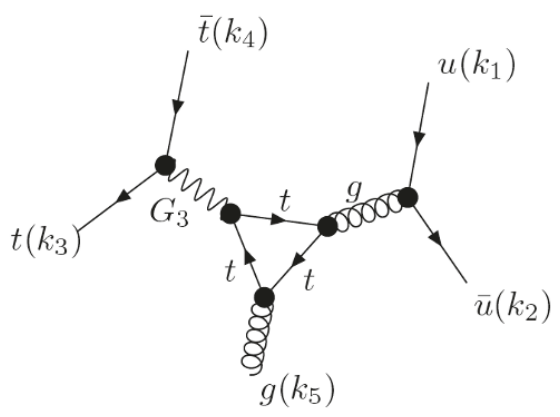

(c)

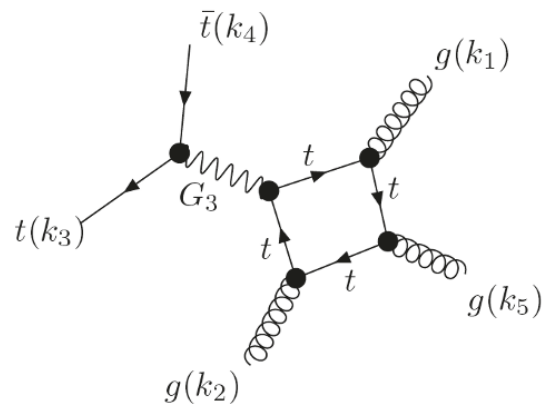

(b)

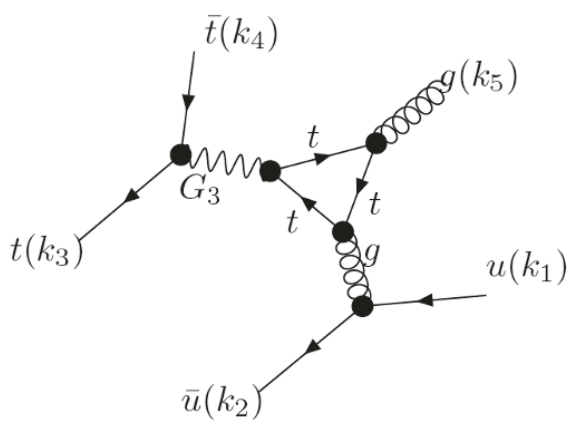

(d)

Figure 8. Loop induced production mode of the $G_{3}(\rightarrow t \bar{t})$ in association with a jet: sample diagrams for the gluon-gluon initial state and the two single diagrams that contribute to the quark initiated subprocess.

and the remaining subprocesses can be obtained by crossing. For the gluon channel $(g g)$, we find 24 diagrams contributing; for the quark channel $(q \bar{q})$, there are only two. The topology for the quark-gluon channel ( $q g$ or $\bar{q} g$ ) is the same as in the $q \bar{q}$ case. Note that in the quark channel, no initial state radiation can occur as these contributions are zero due to vanishing color factors. A sample of gluonic diagrams and the two individual quark initiated diagrams are depicted in figure 8.

In the quark initiated subprocess the only contribution is via an odd number of particles attached to the top quark loop. In case all three particles are vector-like particles, this contribution vanishes due to Furry's theorem. This is the case if $\theta=\pi / 4$, as for this case there is no axial component in the $G_{3}$ resonance. We checked this behavior numerically, which is an important consistency check for our calculation. In the gluon initiated case the contribution does not completely vanish for $\theta=\pi / 4$ as there are still contributions from box diagrams.

Our results are shown in figure 9 at collider energies of $7 \mathrm{TeV}$ and $14 \mathrm{TeV}$ for $c_{t}=1$. Avoiding the chirality suppressed regime $(\theta=\pi / 4)$, these cross sections are comparable to the Higgs boson production cross section over a wide range of $M_{G}$. At $14 \mathrm{TeV}$, they can even become a few times larger. The strong dependence on chirality is noticed especially at $\theta=\pi / 4$, for reasons as explained above. 


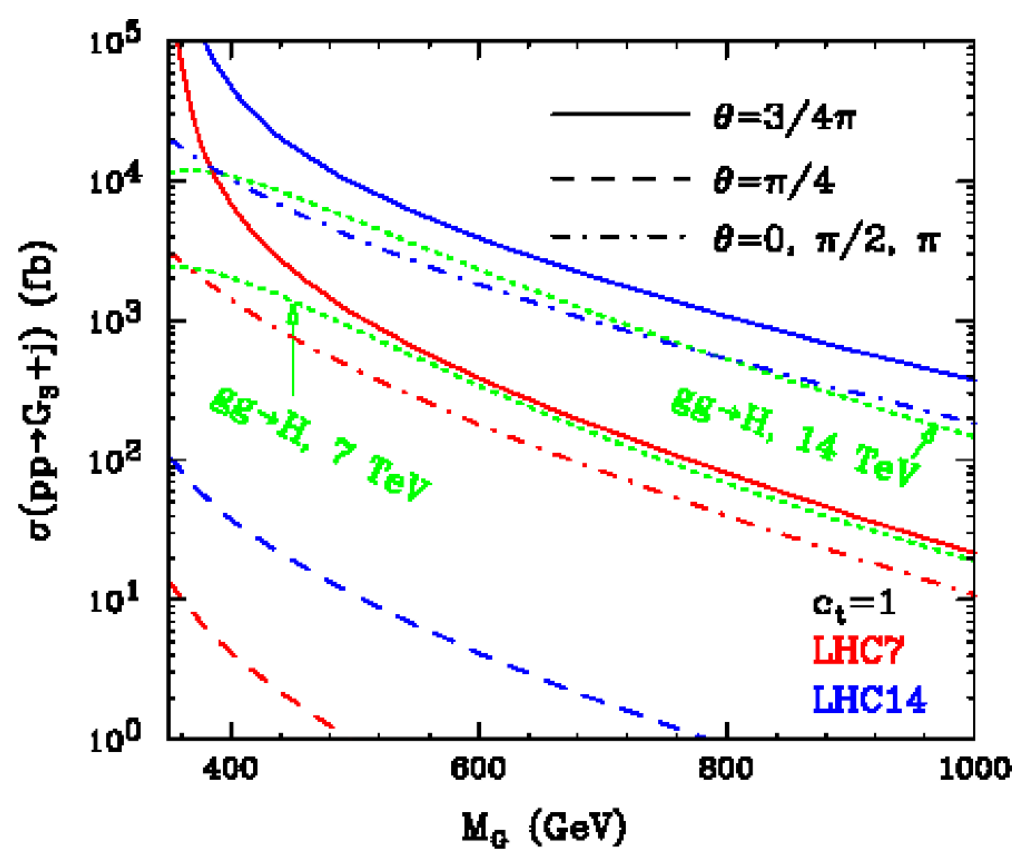

Figure 9. Cross section of the loop induced production mode $p p \rightarrow G_{3}+j$ for various values of $\theta$ and utilizing $c_{t}=1$. The jet defining requirements are given in eq. (2.4). The red and blue curves depict the results for an LHC center-of-mass energy of $7 \mathrm{TeV}$ and $14 \mathrm{TeV}$, respectively. For comparison, the Higgs boson production cross section in gluon fusion with $M_{\mathrm{H}}=M_{G}$ is also shown for both collision energies (cf. the green dotted lines).
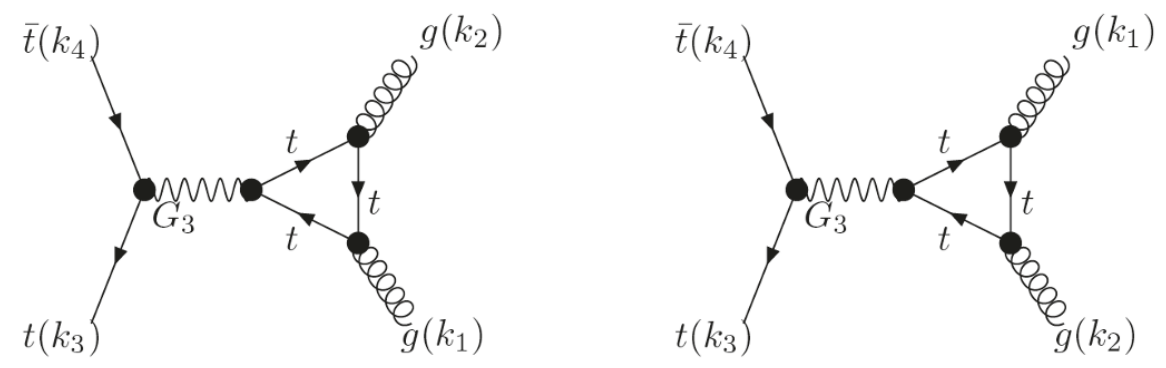

Figure 10. The two diagrams that contribute, at lowest order, to the loop induced production of $G_{3}(\rightarrow t \bar{t})$ plus no extra parton. The quark initiated subprocess vanishes due to the color algebra.

\subsubsection{Off-shell production of the $G_{3}$ resonance: $p p \rightarrow G_{3} \rightarrow t \bar{t}$}

In the case of loop induced, exclusive $G_{3}$ production, all contributions vanish as long as the $G_{3}$ is produced on-shell. This is a consequence of the Landau-Yang theorem. However, there is a non-negligible contribution from the $G_{3}$ being off-shell which gives a non-zero result. Including the $G_{3}$ decay into $t \bar{t}$, we only find two diagrams, which are depicted in figure 10. They each contribute to the gluon initiated subprocess of the $t \bar{t}+0$ jet channel. No other partonic subprocess exists, and we verified numerically that even the $g g$ initial state contribution turns zero in the on-shell case. The latter moreover means that we passed another important check of our calculation. 


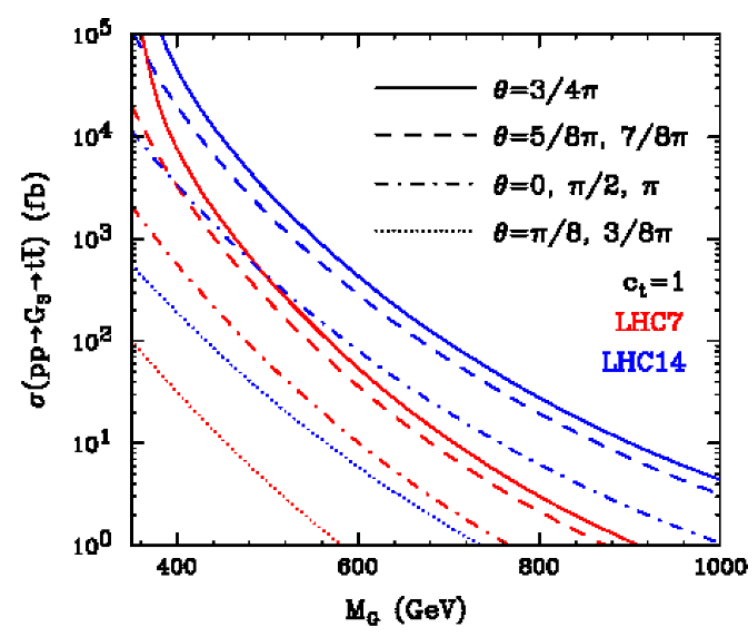

(a)

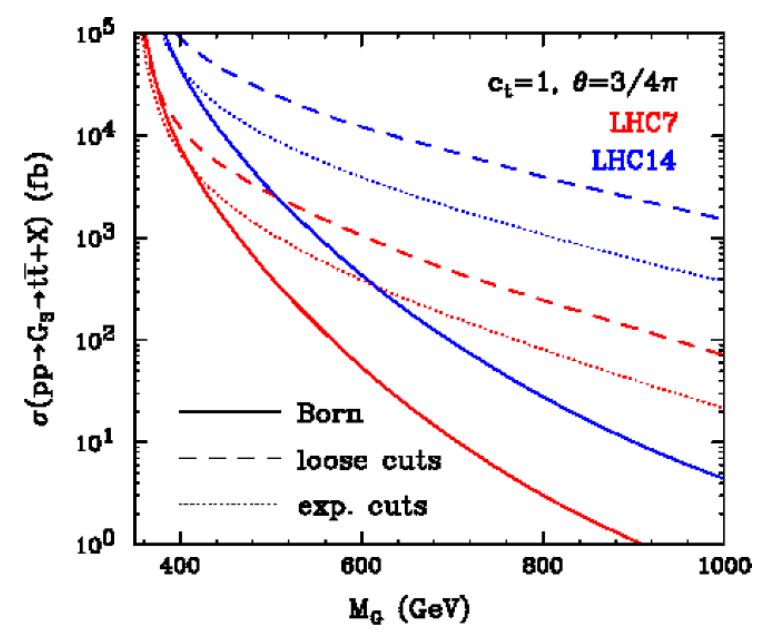

(b)

Figure 11. Production cross section for (11a) the loop induced process $p p \rightarrow G_{3} \rightarrow t \bar{t}$ (the Born process) using $c_{t}=1$ and various values of $\theta$. In the right panel (11b), the maximal cross sections, obtained by choosing $\theta=\frac{3}{4} \pi$, are compared to those for the loop induced process with an additional parton in the final state. While the "experimental cuts" are given in eq. (2.4), the "loose cuts" are defined by $p_{T, j}>20 \mathrm{GeV}$ and a fairly wide jet rapidity window of $\left|\eta_{j}\right|<6.0$.

Figure 11a can be directly compared to figure 9 as it uses the same layout to show the cross section dependence on the mass of the $G_{3}$ particle for our choice of $c_{t}=1$ and several different values of the chirality angle $\theta$. As can be seen from these figures, one important consequence of the model - which at first seems unusual - is the sizeable increase of the cross section for associated jet production with respect to that of the pure $G_{3} \rightarrow t \bar{t}$ process. Their relative importance strongly depends on the mass of the $G_{3}$, and varies from being roughly of the same size for $M_{G}=400 \mathrm{GeV}$ up to about two orders of magnitude for $M_{G}=1 \mathrm{TeV}$. This is nicely demonstrated in figure $11 \mathrm{~b}$ for the choice of $\theta=3 \pi / 4$, which maximizes the cross section in both cases.

One reason for the enhancement of the $t \bar{t}+$ jet channel is the appearance of box diagrams in the $t \bar{t}+1$ parton processes. These box diagrams are not governed by Furry's theorem owing to the even number of spin-1 particles attached to the loop. Another reason is that initial state radiation can shift the gluon attached to a triangle off-shell, thus enabling the $G_{3}$ to go on-shell. It is therefore expected that the $G_{3} j$ associated production will contribute substantially to the inclusive cross section determination based on $t \bar{t}$ final states. Moreover, even for an exclusive $t \bar{t}+0$ jet measurement, there will be a contribution stemming from the case where the jet is not resolved, and this contribution cannot be neglected in the zero-jet bin. Because of these facts, we will have to combine the parton level calculations for $G_{3}+0$ and $G_{3}+1$ extra parton, to arrive at an "approximate NLO" prediction. Only after we can use this prediction to apply the cross section bounds from inclusive and exclusive $t \bar{t}+0$ jet measurements to constrain the $G_{3}$ model parameters. We return to this discussion in more detail in the next section. 


\section{Current experimental bounds and future prospects for LHC Run 2}

We will use results from searches in multi-top quark final states and recent experimental measurements related to top quark pair production, also in association with jets, to constrain the parameter space (mass and couplings or chirality) for the production of the $G_{3}$ resonance. Cross section limits are among the strongest handles that we can utilize to extract current bounds from the Tevatron as well as from the LHC for 7 and $8 \mathrm{TeV}$ collision energies. Taken these bounds, we have to make sure to choose the model parameters such that our signal evades all of these bounds; only after we can discuss the prospects of the model we are considering here for resonance searches at the $14 \mathrm{TeV}$ LHC.

If we compare the cross sections between the tree level and one-loop production modes, we notice a clear ranking in favor of the loop induced modes. Roughly speaking, they are separated by at least two orders of magnitude for light $G_{3}$ resonances $\left(M_{G} \approx 400 \mathrm{GeV}\right)$ where we obtain $\mathcal{O}(10 \mathrm{pb})$ cross sections in the loop induced production channels. For the heavier $G_{3}$ (with $M_{G} \approx 1 \mathrm{TeV}$ ), we find the $G_{3} j$ one-loop mode dominating over all other channels by a factor of about 50 . The pure $G_{3}$ mode drops much faster and becomes comparable to the production at the tree level, which is of the order of $10 \mathrm{fb}$ for LHC $14 \mathrm{TeV}$ but remains well below $1 \mathrm{fb}$ for LHC Run 1 energies. The loop induced modes are therefore essential in a search for top-philic resonances, especially for a test of the high mass regime where these modes become indispensable. This does not mean that we should discard the tree level production channels right away. They give rise to more exotic final states characterized by multi-top quark signatures that do not have to compete with the larger backgrounds, which occur for loop level generated $\left(G_{3} \rightarrow\right) t \bar{t}+0,1$ jet final states. Because of their peculiar signatures, the tree level modes carry valuable information and provide a great opportunity for diversifying the search, in particular for low mass top-philic resonances.

\subsection{Bounds from searches for multiple top quark final states}

All tree level production modes lead to either three top quarks $\left(G_{3} t j\right.$ and $\left.G_{3} t W\right)$ or four top quarks $\left(G_{3} t \bar{t}\right)$ in the final state. This prominent feature of abundant top quarks needs to be exploited in searching for distinct signs of decaying $G_{3}$ resonances.

In particular the largest production channel at the tree level, the $G_{3} t \bar{t}$ process results in a final state with four top quarks, which is constrained by a CMS study [44]. ${ }^{2}$ The CMS upper limit on the four top-quark production cross section is $32 \mathrm{fb}$ (95\% C.L.) at LHC $8 \mathrm{TeV}$ with $19.6 \mathrm{fb}^{-1}$. This constraint is depicted in the top left corner of figure 12 by the area that has been shaded in plain yellow. The (other) contours in the $M_{G}-c_{L}$ parameter plane where $c_{L} \equiv c_{R}=c_{t} / \sqrt{2}$ represent the production cross section of four top quarks at an LHC energy of $14 \mathrm{TeV} .^{3}$ From this visualization, we note that most of the parameter space remains unconstrained, yet becomes conveniently testable at Run 2 for rather natural choices of the coupling $\left(c_{L} \sim \mathcal{O}(1) \sim c_{R}\right)$ and $G_{3}$ masses below $\sim 1000 \mathrm{GeV}$.

\footnotetext{
${ }^{2}$ Similar studies have been carried out by ATLAS providing a weaker limit for the $7 \mathrm{TeV}$ LHC [45].

${ }^{3}$ As discussed in section 2.2 , the chirality $(\theta)$ dependence of the cross section is very weak, calling for the obvious choice to show the cross section as a function of the model parameters $M_{G}$ and $c_{t}$.
} 


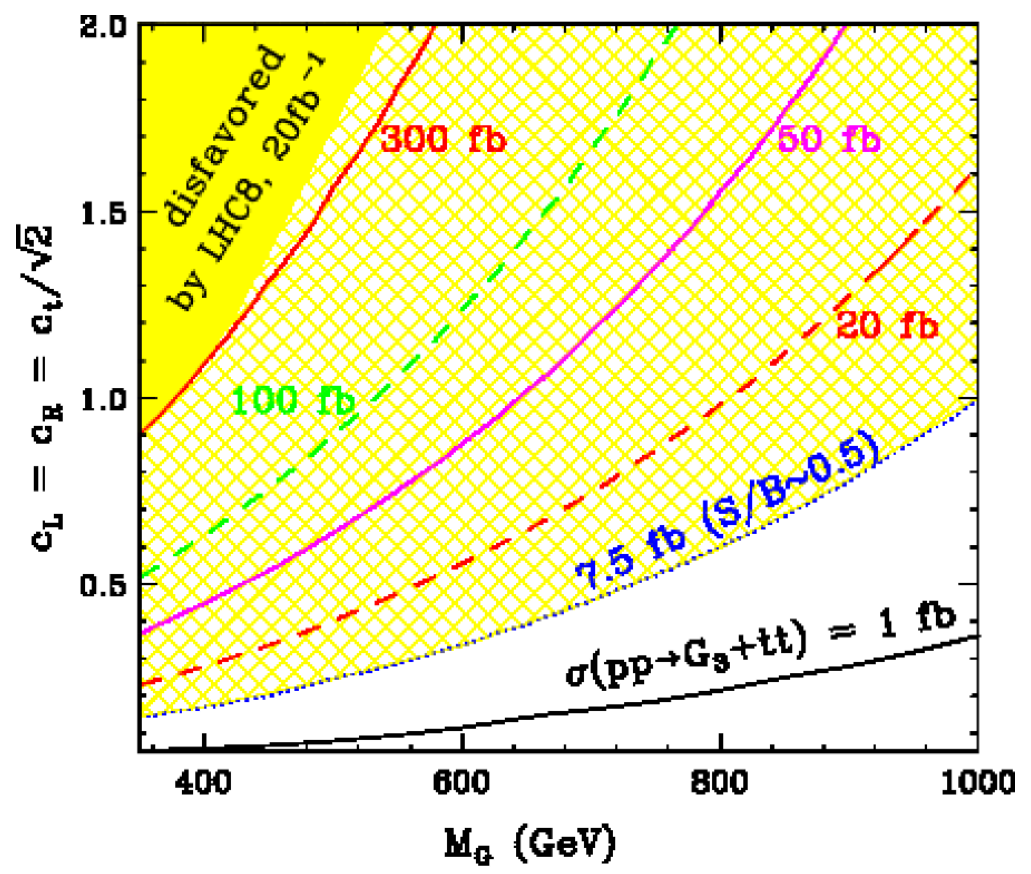

Figure 12. Production cross section at the $14 \mathrm{TeV}$ LHC for the tree level process $p p \rightarrow G_{3}+t \bar{t}$ shown in the model parameter plane of $M_{G}-c_{t} / \sqrt{2}$. The upper left corner (shaded in yellow) of this parameter space has been already probed by the $8 \mathrm{TeV}$ LHC. The lighter shade is used to indicate an exclusion potential that may be achieved for Run 2 of the LHC.

At $14 \mathrm{TeV}$ the SM prediction for four top quarks at NLO is around $15 \mathrm{fb}$ (see ref. [46]). This means that the contribution from the $G_{3}$ signal can lead to a significant enhancement of the four top-quark cross section yielding a large signal over background ratio $(S / B)$ for the major fraction of the parameter space. If we take the scale variation as a measure for the systematic uncertainty of the NLO calculation, which the authors of ref. [46] determined to be around $\pm 4 \mathrm{fb}$, the measurement in the four top-quark channel will unavoidably be dominated by systematic uncertainties. A meaningful search thus requires a large enough $S / B$ of around 0.5 . This yields a rough estimate for the potential of a Run 2 search, which we mark in figure 12 using the lighter shade of yellow. Note that the $S / B$ requirement is more stringent, as the statistical significance $(S / \sqrt{B})$ for the discovery of a potential signal of $7.5 \mathrm{fb}$ can be easily reached with as little as $7 \mathrm{fb}^{-1}$ of data.

\subsection{Bounds from uncertainties in top quark pair production measurements}

The one-loop production modes can be traced in a very general way through their $t \bar{t}+$ jets signatures. In both cases considered here, the $G_{3}$ vector particles decay into rather energetic top quark pairs, which will likely be accompanied by one jet owing to the dominance of the $G_{3} j$ production channel. This is true in particular for heavy resonances above $M_{G}=600 \mathrm{GeV}$, cf. figure 11b. It is of course no secret that $t \bar{t}+$ jets final states are populated by a fairly large number of background processes but from experimental measurements designed to track down $t \bar{t}$ pairs, we already have a great deal of experience to which kinematical requirements work best in such an environment. In our case, we thus will be 
confronted with SM top quark pair production as the by far largest contribution to the portfolio of backgrounds. At zeroth order, we hence are in the position to neglect the other processes such as $W+$ jets, and just rely on the excellent theoretical and experimental control of the $t \bar{t}$ production [47] to obtain reasonable estimates for the detection of the imprints from decaying $G_{3}$ resonances.

Considering the production of $t \bar{t}$ final states, we have to bear in mind that they may emerge in association with a number of $X$ jets, denoted conveniently by $t \bar{t}+X$. In a fully inclusive measurement, all jet contributions, $X=0,1, \ldots$, are taken into account while for an exclusive $t \bar{t}+X$ analysis, one requires to find exactly $X$ jets accompanying the top quark pair. In general there are three different levels of experimental exclusiveness which are roughly characterized by (1) inclusive total $t \bar{t}$ cross section measurements, (2) differential measurements in the number of jets associated with the $t \bar{t}$ production and (3) measurements of differential distributions based on pair and single top quark properties such as the invariant mass or the transverse momentum. Here, we will exploit the first two categories only, leaving us with plenty of data from which we can infer important constraints on the $G_{3}$ model. To distinguish them more easily in our discussion, we introduce the following notation: "(I)" stands for the inclusive measurements of point (1) while "(E)" denotes the exclusive ones under point (2).

The third lever arm that we could utilize is given by differential distributions based on $t \bar{t}$ system or single top quark objects. Related observables can then be used to check on individual bins, which should better be unaffected by signal contributions, at least beyond a two-sigma deviation. However, these investigations are outside the scope of this paper and their outcome will be discussed in a forthcoming publication.

\subsubsection{Inclusive jet cross sections}

Measurements in the two lowest $t \bar{t}+\geq X$ jet bins are of importance to our discussion, namely the production of $t \bar{t}$ final states plus any number of jets $(t \bar{t}+\geq 0$ jets), which we label "(I0)", and those occurring in association with at least one jet $(t \bar{t}+\geq 1$ jet), which we label "(I1)". Accordingly, we can extract two types of limits from the respective uncertainties on the cross section measurements: that is the inclusive zero-jet limit and the inclusive one-jet limit, which we denote $f \times \Delta \sigma_{t \bar{t}}^{(\geq 0)}$ and $f \times \Delta \sigma_{t \bar{t}}^{(\geq 1)}$, respectively. The factor $f$ indicates that we have some leeway in applying these limits. We certainly are on the safe side with factors of about two, allowing the signal to grow no more than into a two-sigma deviation.

Focusing first on the entirely inclusive scenario (I0), we have to confront the zero-jet limit with a prediction for the loop induced production of $G_{3}$ that is fully accurate at NLO in QCD. In a rigorous approach, this order of precision is forced upon us because of the largeness of the one-jet contribution with respect to the contribution obtained at the Born level. This peculiarity of our signal model has been demonstrated in figure $11 \mathrm{~b}$ but it may also be understood as a strong motivation to calculate the two-loop corrections to our $G_{3}$ signal (for which we use the notation " $S$ " here). A two-loop accurate treatment however is not within the scope of this paper and we have to resort to an alternative. Knowing that 
a signal cross section description via

$$
\sigma_{S, \mathrm{LO}}^{(\geq 0)}=\sigma_{\mathrm{Born}} \quad \text { or } \quad \sigma_{S, \mathrm{NLO}}^{(\geq 0)}=\sigma_{\mathrm{Born}}+\sigma_{\mathrm{Virt}}+\sigma_{\text {Real }}
$$

cannot be trusted or remains, respectively, out of reach for now, we sign up for a compromise between the two, which we will refer to as an approximate NLO treatment of the signal.

We can write the signal cross section contributing to the inclusive zero-jet bin as

$$
\sigma_{S, \mathrm{NLO}}^{(\geq 0)}=\sigma_{\text {Born }}+\sigma_{\text {Real }}^{\text {loose cuts }}+\delta \leq f \times \Delta \sigma_{t \bar{t}}^{(\geq 0)}
$$

where we use

$$
\delta=\sigma_{\text {Virt }}+\sigma_{\text {Real }}^{\mathrm{IR}}
$$

to collect all terms, which cannot be calculated within the scope of this paper. Note that eq. (3.2) already shows the bound to the signal inclusive $t \bar{t}$ cross section. To motivate, moreover justify our simplified approach, it is useful to consider the different regions of the $p_{T, t \bar{t}}$ distribution. The Born term which we calculate from the $t \bar{t}+0$ parton amplitudes (at LO) contributes to the $p_{T, t \bar{t}}=0$ bin, which also receives virtual corrections as accounted for by $\sigma_{\text {Virt }}$ occurring in the $\delta$ term. The real-correction term in eq. (3.2) again can be calculated (at LO), this time from the $t \bar{t}+1$ parton amplitudes. The label "loose cuts" is chosen to express a careful separation between the fixed-order and resummation $p_{T, t \bar{t}}$ regime that can be obtained by applying rather loose jet cuts, which are more inclusive than common experimental jet definitions, yet safe enough to ensure a meaningful LO description of the $p_{T, t \bar{t}}$ spectrum above the Sudakov region (i.e. for $p_{T, t \bar{t}} \gtrsim 20 \mathrm{GeV}$ ). The Sudakov region itself cannot be treated without the proper inclusion of resummation effects below $p_{T, t \bar{t}} \approx 20 \mathrm{GeV}$. The $\operatorname{soft}\left(-p_{T, t \bar{t}}\right)$ real-correction term, $\sigma_{\text {Real }}^{\mathrm{IR}}$, is therefore part of the $\delta$ correction to $\sigma_{S, \mathrm{NLO}}^{(\geq 0)}$. Of course, $\delta$ is separately finite by construction as it contains (and cancels out) all IR divergences arising from the virtual as well as the real corrections.

It helps our discussion to re-order eq. (3.2) as follows:

$$
\left(\frac{\sigma_{\text {Born }}+\sigma_{\text {Real }}^{\text {loose cuts }}}{\Delta \sigma_{t \bar{t}}^{(\geq 0)}}\right) \times\left(1+\frac{\delta}{\sigma_{\text {Born }}+\sigma_{\text {Real }}^{\text {loose }}}\right) \leq f .
$$

The first bracket on the left-hand side is now fully controlled by our parton level calculations, and we can individually constrain it, for example, to be smaller than two. For the second bracket, it is not unreasonable to assume that its cross section ratio varies no more than $\pm 50 \%$, or very conservatively $\pm 100 \%$. This leaves us with an estimate for the left-hand side of eq. (3.4) reading $2 \times(1 \pm 0.5)=1, \ldots, 3 \leq f$, which is completely within the cross section range that cannot be excluded owing to the measurement uncertainties. Thus, although we are forced to speculate by and large about the size of the (unknown) $\delta$ contribution, we conclude that in the realm of (I0) analyses it is sufficiently safe to set bounds according to

$$
\sigma_{\text {Born }}+\sigma_{\text {Real }}^{\text {loose cuts }} \leq f \times \Delta \sigma_{t \bar{t}}^{(\geq 0)} .
$$

This means, instead of constraining the Born cross section only, the inclusive zero-jet limit has to be used to constrain the summed up cross section for both the $t \bar{t}+0$ and $t \bar{t}+1$ 
parton calculations. The looser phase-space definition of the jet in the one-parton process enables us to include some of the soft $p_{T, t \bar{t}}$ contributions that usually remain unresolvable in a realistic jet analysis, even though we are clearly not in the position to provide a full NLO treatment of the signal.

Turning to the discussion of the inclusive one-jet limits (I1), we immediately recognize that an approximate NLO treatment for $G_{3} j$ would require us to compute the double-real corrections to the loop level generation of $G_{3}$. As for the two-loop case, we leave this calculation to a future study; also because we only expect moderate real corrections to the $G_{3} j$ rate, which should be much smaller than the drastic corrections that we found for the $G_{3}$ rate. This assumption should be reasonable since there is no mechanism in place, based on Furry's theorem, that could suppress the Born level of the $G_{3} j$ process in a way comparable to the suppression of the $G_{3}$ Born process. Based on these arguments, it is absolutely sufficient to directly apply the one-jet limit to the $t \bar{t}+1$ parton (LO) calculations obeying (as closely as possible) the jet requirements as given by the particular experiment. Hence, we can summarize this situation as

$$
\sigma_{S, \mathrm{LO}}^{(\geq 1)}=\sigma_{\text {Real }}^{\text {exp. cuts }} \leq f \times \Delta \sigma_{t \bar{t}}^{(\geq 1)}
$$

noting that $\sigma_{\text {Real }}^{\text {exp. cuts }}<\sigma_{\text {Real }}^{\text {loose cuts }}$ by construction - in other words, the use of experimental cuts pushes us further out in $p_{T, t \bar{t}}$, i.e. further away from the Sudakov region in the $p_{T, t \bar{t}}$ distribution as compared to the zero-jet case.

\subsubsection{Exclusive jet cross sections and the $N_{\text {jets }}$ distribution}

In contrast to the previously discussed situation, differential distributions in the number of jets, in short $N_{\text {jets }}$ distributions, give us the opportunity to extract cross section limits for exclusive $t \bar{t}+X$ final states. We denote these quantities by $\Delta \sigma_{t \bar{t}}^{(X)}$ as opposed to $\Delta \sigma_{t \bar{t}}^{(\geq X)}$, which are used to express the cross section uncertainties of the inclusive jet bins. Again, the measurements of interest to us are those targeting the $t \bar{t}+0$ jet bin (no extra-jet bin) and the $t \bar{t}+1$ jet bin (exactly one-jet bin), which help us obtain estimates for the exclusive zero-jet and one-jet limits, $\Delta \sigma_{t \bar{t}}^{(0)}$ and $\Delta \sigma_{t \bar{t}}^{(1)}$, respectively. Within our labelling scheme, we distinguish these two cases by "(E0)" and "(E1)".

Based on our findings for the (I0) case, we can easily derive an NLO approximate relation that obeys the kinematical requirements on (E0) final states. To incorporate the zero-jet exclusiveness, we modify eq. (3.5) by removing the resolved-jet contribution

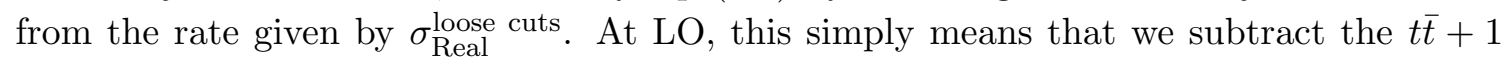
parton cross section for experimental jet cuts from the left-hand side of eq. (3.5). In the (E0) category, we therefore only constrain the leftover part, i.e. the unresolved part of the respective (I0) relation, and hence arrive at

$$
\sigma_{\text {Born }}+\sigma_{\text {Real }}^{\text {loose cuts }}-\sigma_{\text {Real }}^{\text {exp. cuts }} \leq f \times \Delta \sigma_{t \bar{t}}^{(0)} .
$$

Note that there are no changes to the loose cut definition; the loose jet constraints are chosen as before to keep the resummation effects negligible. 
Similarly to (I1), any exclusive one-jet limit can be used to place bounds on the $t \bar{t}$ plus one-parton cross section. Already in the context of a leading-order calculation, this is a reasonable approach for the same reasons as previously described for the case of (I1). All we need to constrain is the resolved contribution of $\sigma_{\text {Real }}^{\text {loose cuts }}$, and practically there is no difference whether we consider (E1) or (I1) as long as the $t \bar{t}+1$ parton calculation has no more than LO accuracy. Equation (3.6) therefore shows almost no difference to what we write down for the case of (E1) where we have

$$
\sigma_{\text {Real }}^{\text {exp. cuts }} \leq f \times \Delta \sigma_{t \bar{t}}^{(1)} .
$$

The difference between this LO expression and eq. (3.6) originates merely from the specifics of the (experimental) jet definition applied in both the exclusive and inclusive scenarios. These kinematical choices determine whether the extra parton gets "resolved" as a jet or missed in the bulk of soft/collinear radiation.

Exclusive jet cross sections are rarely reported directly. We have to infer them from $N_{\text {jets }}$ distributions, usually presented in terms of exclusive $X$-jet fractions for $\ell+$ jets and dilepton final states confined to a fiducial volume. In some cases, these jet fractions, $r_{X}$, are also provided for the distribution of additional jets occurring in $t \bar{t}+X$ production. The computation of an exclusive $X$-jet cross section is thus expressed as

$$
\sigma_{t \bar{t}}^{(X)}=r_{X} \times \sigma_{t \bar{t}}^{(\geq 0)}
$$

and requires knowledge of $\sigma_{t \bar{t}}^{(\geq 0)}$, the total inclusive rate for $t \bar{t}$ production, which can be taken from flagship measurements that release this cross section after the application of unfolding and acceptance corrections. The uncertainty associated with the exclusive jet cross sections is hence given as

$$
\Delta \sigma_{t \bar{t}}^{(X)}=\left(\frac{\Delta r_{X}}{r_{X}}+\frac{\Delta \sigma_{t \bar{t}}^{(\geq 0)}}{\sigma_{t \bar{t}}^{(\geq 0)}}\right) \times r_{X} \times \sigma_{t \bar{t}}^{(\geq 0)},
$$

which we have to take into account when we determine the cross section limits in the next subsection. Note that eq. (3.10) is written for the more conservative approach of summing the two individual uncertainties linearly rather than adding them in quadrature as one does for uncorrelated Gaussian errors.

\subsubsection{Constraints on the loop level resonance production}

Given the four types of limits discussed above, we worked out how to apply them to our specific calculations. We now need to quantify the exact size of the inclusive and exclusive cross section uncertainties $\Delta \sigma_{t \bar{t}}^{(\geq X)}$ and $\Delta \sigma_{t \bar{t}}^{(X)}$, respectively, using current experimental results. Table 1 lists the values, which we determined in order to make the relations (3.5), (3.6), (3.7) and (3.8) explicit. As explained before, we argued for $f=2$ as a safe choice in fixing the $f$-factor. Our results however will be reported for the more restrictive case of using $f=1$.

The Tevatron limits in table 1 have been obtained, for (I0), from the combination of measurements with the goal to determine the top quark pair production cross section at 


\begin{tabular}{|lrcccc|}
\hline Collider & Category: & I0 & I1 & E0 & E1 \\
\hline Tevatron $1.96 \mathrm{TeV}$ & 0.41 & 0.54 & 0.81 & & \\
LHC $7 \mathrm{TeV}$ & 10 & 26 & $8, \ldots, 9$ & 12 & \\
LHC $8 \mathrm{TeV}$ & 13 & & & & \\
\hline
\end{tabular}

Table 1. Overview of the $\Delta \sigma_{t \bar{t}}$ quantities, in $\mathrm{pb}$, for the different cross section limit categories concerning current inclusive (IX) and exclusive $(\mathrm{E} X)$ cross section measurements for $t \bar{t}$ production in association with $X=0,1$ jets.

$1.96 \mathrm{TeV}$ and, for (I1) and (E0), from a CDF measurement of the $t \bar{t}+$ jet cross section with $4.1 \mathrm{fb}^{-1}$ of Tevatron data. The results and their related uncertainties have been reported in refs. [48] and [49], respectively. We have checked that these limits are of no consequence for the hadronic production of the $G_{3}$ as the gluon initiated subprocesses cannot be tightly constrained at the Tevatron. As a matter of fact, to cross (or reach) the production threshold of $t \bar{t}$ pairs, the Tevatron was forced into the operational mode of a $q \bar{q}$ collider, leaving obviously little room to test the highly important gluon production channels of the $G_{3}$ model. In contrast to the Tevatron, the LHC predominantly operates as a $g g$ collider. It is the more natural place to look for $G_{3}$ resonances and therefore enables us to set stronger limits on $\left(G_{3}\right.$ induced) deviations from SM $t \bar{t}$ production. Thus, all other cross section limits shown in table 1 have been extracted from a variety of LHC measurements.

Considering the most inclusive, the (I0) case first, we have several comparable cross section measurements from the ATLAS and CMS collaborations for both Run 1 energies, in the single lepton plus jets $(\ell+$ jets $)$ channel as well as the dilepton $(\ell \ell)$ channel $[50-56,60]$. For the $7 \mathrm{TeV}$ LHC, we find the CMS dilepton measurement [51] based on $\mathcal{L}=2.3 \mathrm{fb}^{-1}$ to be very accurate, but as a result of the large spread among the different central values of all measurements, we have decided for a reasonably safe compromise, which is to use the $10 \mathrm{pb}$ uncertainty of the LHC combined result published in September 2012 [57]. For the $8 \mathrm{TeV}$ LHC (and an $m_{t}$ reference value of $172.5 \mathrm{GeV}$ ), the top quark pair production cross section has been determined very recently as $\sigma_{t \bar{t}}=(241.5 \pm 8.5) \mathrm{pb}$, with an additional uncertainty of $4.2 \mathrm{pb}$ owing to LHC beam effects [58]. This is the result of a first combination of ATLAS and CMS measurements with exactly one electron and one muon in the final state, which benefits from the fact that both the ATLAS and CMS dilepton channels have delivered very precise results with uncertainties marginally larger than $10 \mathrm{pb}[53,59,60]$. Again, the spread in all $8 \mathrm{TeV}$ measurements is at least of similar size, and hence we have chosen, as before, to be more conservative in our table where we included the somewhat larger uncertainty $(13 \mathrm{pb})$ of the CMS dilepton channel obtained after analyzing $5.3 \mathrm{fb}^{-1}$ of data [60].

From a theoretical point of view, it is interesting to note that the (I0) numbers cannot be controlled more precisely than they have been actually measured in the experiments. The current situation is described adequately by the fact that experimental and theoretical uncertainties are of the same size. This becomes clear by comparing the (I0) limits in table 1 with the total theory errors $\left(\delta_{\text {tot }}\right)$ on $\sigma_{t \bar{t}}$ as published in refs. [47, 61] for Tevatron Run II and LHC Run 1 energies. 
For the other jet categories, (I1), (E0) and (E1), it is more intricate to find suitable results, which work on the level of top quarks and additional jets. This level of information requires several steps of data corrections such as the unfolding of detector effects, the kinematic reconstruction of top quarks and the extrapolation from the acceptance region to the full phase space. These experimental procedures are sophisticated, costly, and take a lot of work. Still, there are a number of publications (and more will become available with time), in particular for the $7 \mathrm{TeV}$ LHC, that help us specify the limits for the categories other than (I0).

In ref. [62], the cross section for the production of $t \bar{t}$ pairs in association with at least one jet has been reported for the $7 \mathrm{TeV}$ LHC. The measurement results are given in a fiducial volume as well as in the inclusive one-jet phase space defined by the anti- $k_{T}$ jet algorithm. The latter, i.e. the acceptance-corrected result is used to assign a value to $\Delta \sigma_{t \bar{t}}^{(\geq 1)}$ as shown in table 1. Concerning our calculations, we have then made sure that the jet parameters of eq. (2.4) like $p_{T, j}, \eta_{j}$ and $R$ exactly match those used by the experiment.

The (E0) and (E1) $7 \mathrm{TeV}$ limits have been obtained from the results given in section 7 of the CMS publication on jet multiplicity distributions [63]. Their jet finding procedure is again based on the anti- $k_{T}$ algorithm, this time with slightly different parameter settings reading $p_{T, j}>30 \mathrm{GeV},\left|\eta_{j}\right|<2.4$ and $R=0.5$. The $N_{\text {jets }}$ distribution however is also provided in terms of additional jets on top of the already reconstructed $t \bar{t}$ system. ${ }^{4}$ This is a big advantage for us as it avoids any discussion concerning the top quark decay effects that we cannot simulate here for the $G_{3}$ signal. Without this reconstruction, the $G_{3}$ production particularly affects the $2+3$-jet and the $4+5$-jet bins of the $\ell \ell$ and the $\ell+$ jets channel, respectively, however in a complicated way that is hard to approximate without the proper implementation of the decays. As we learn from the results of ref. [63] (cf. figure 4 therein), using an additional jet counting, little of the zero-jet contribution is found in the 5-jet bin of the $\ell+$ jets channel where the decay jets are included to the jet counting. The $t \bar{t}+1$ jet contribution however spreads rather equally over the 4-jet and 5-jet bin. It is not clear a priori whether the $G_{3}$ signal including its top quark decays will leave an imprint similar to that given by ordinary $t \bar{t}$ production. This emphasizes the importance of the CMS $t \bar{t}+$ jets data (given in table 4 and figure 6 ) of ref. [63]. For the exclusive jet fractions, $r_{X}$, we find $r_{0}=0.332$ and $r_{1}=0.436$ quoted with an uncertainty of $9.0 \%$ and $9.8 \%$, respectively. Based on eq. (3.10), we then obtain the upper limits on the signal exclusive-jet cross sections: $\Delta \sigma_{t \bar{t}}^{(0)}=8.5 \mathrm{pb}$ and $\Delta \sigma_{t \bar{t}}^{(1)}=11.8 \mathrm{pb}$ where we used $\sigma_{t \bar{t}}^{(\geq 0)}=(173 \pm 10) \mathrm{pb}$ in accordance with our choice for the (I0) case. ${ }^{5}$ There is one caveat though; the results stated by CMS are strictly valid only for a visible phase-space definition (as clearly described in the beginning of section 6 of ref. [63]). This visible phase-space definition includes certain kinematical

\footnotetext{
${ }^{4}$ The corresponding ATLAS publication [64] as well as its earlier conference note [65] do not contain $N_{\text {jets }}$ information at the level of reconstructed top quarks and jets. In comparison to ref. [63] (cf. table 3 therein), we nevertheless use the $N_{\text {jets }}$ distributions provided by ATLAS in the $\ell+$ jets channel to verify that both collaborations indeed find similar sizes in the error accounting for the lower jet bins. However, we do not expect improvements to the limits derived from the CMS measurement as the uncertainties stated by ATLAS turn out to be somewhat larger.

${ }^{5}$ The more aggressive, i.e. quadrature summed version of eq. (3.10) would yield slightly smaller exclusive jet cross section limits: $\Delta \sigma_{t \bar{t}}^{(0)}=6.1 \mathrm{pb}$ and $\Delta \sigma_{t \bar{t}}^{(1)}=8.6 \mathrm{pb}$.
} 


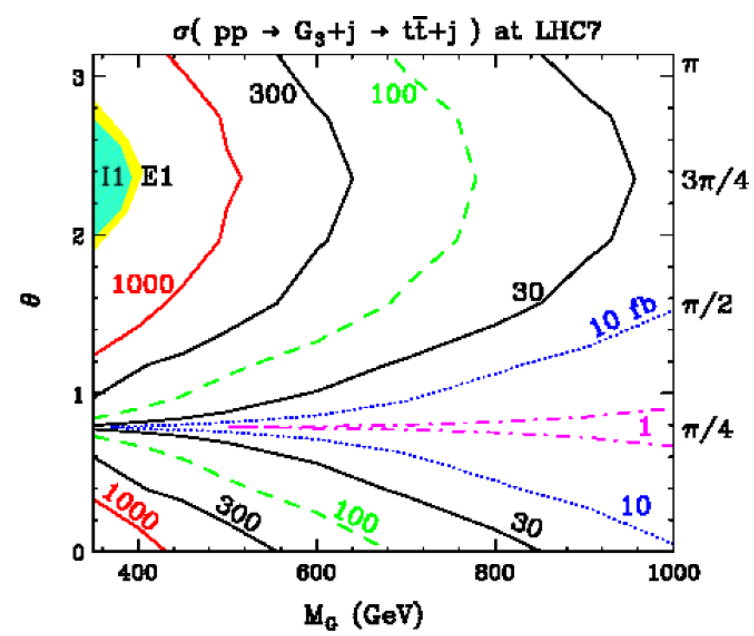

(a)

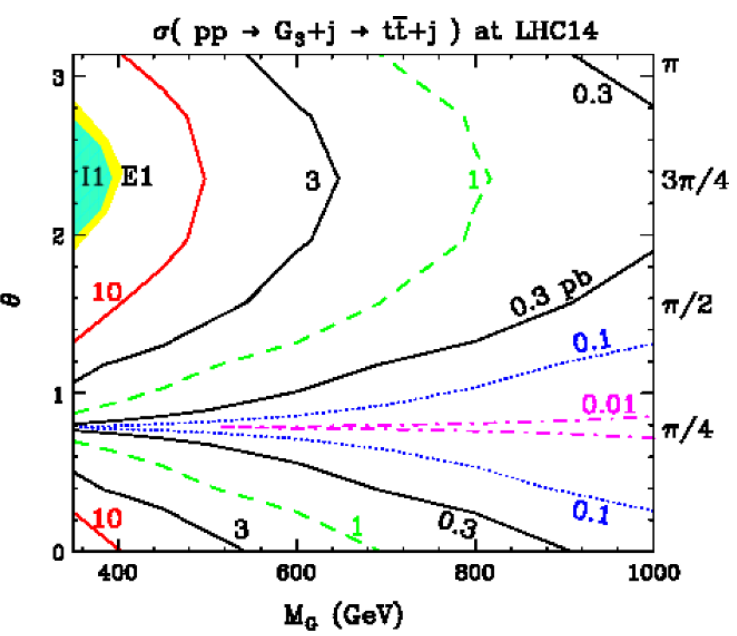

(b)

Figure 13. Lines of constant production cross section in the $M_{G}-\theta$ parameter plane using $c_{t}=1$ for the loop induced processes $p p \rightarrow G_{3}+j \rightarrow t \bar{t}+j$ at proton-proton colliders of $7 \mathrm{TeV}$ (13a) and $14 \mathrm{TeV}$ (13b) center-of-mass energy. Results have been obtained employing the experimental jet cuts as summarized in eq. (2.4). The excluded parameter space, at the one-sigma level, is indicated by the shaded areas (using the labels of table 1), with more explanations given in the text.

requirements on the top quark decay products that we cannot implement here. In a first approximation, it is however not unrealistic to assume that the CMS results carry over to the full phase space without taking any corrections into account.

For the $8 \mathrm{TeV}$ LHC, we did find some useful information in ref. [66], which however is not quite sufficient to evaluate reasonable constraints that apply to the more exclusive jet bin selections (I1), (E0) and (E1). We would need to make a number of assumptions, in particular regarding acceptance corrections and the kinematic effect of top quark decays, which eventually turns the whole procedure of determining limits into a more or less speculative exercise. To our knowledge, direct results of the type "top quarks plus jets" at $8 \mathrm{TeV}$ are in the pipeline; however they have not been published yet. It furthermore remains an open question whether such new $8 \mathrm{TeV}$ limits could be immediately competitive with those extracted from the $7 \mathrm{TeV}$ LHC measurements.

We start the discussion on parameter constraints with the $t \bar{t}+1 j$ case. Figure 13 shows the lines of constant cross sections in a plane spanned by the model parameters $M_{G}$ and $\theta$. The left plot shows the results for $E_{\mathrm{cm}}=7 \mathrm{TeV}$ whereas the plot on the right-hand side contains the predictions for $E_{\mathrm{cm}}=14 \mathrm{TeV}$. Using eq. (3.6) with $f=1$, we indicate cross sections that are excluded at $7 \mathrm{TeV}$ at the one-sigma level. The green shaded area shows the region in parameter space which is excluded by an inclusive one-jet measurement (see (I1) in table 1). The more constraining yellow area displays the limit from exclusive one-jet measurements, according to (E1). While in the $7 \mathrm{TeV}$ plot the border of the shaded area corresponds to a line of constant cross section, this does not have to be the case for the $14 \mathrm{TeV}$ plot. Here the shaded area only marks a region in parameter space that is already excluded by the $7 \mathrm{TeV}$ measurement. 


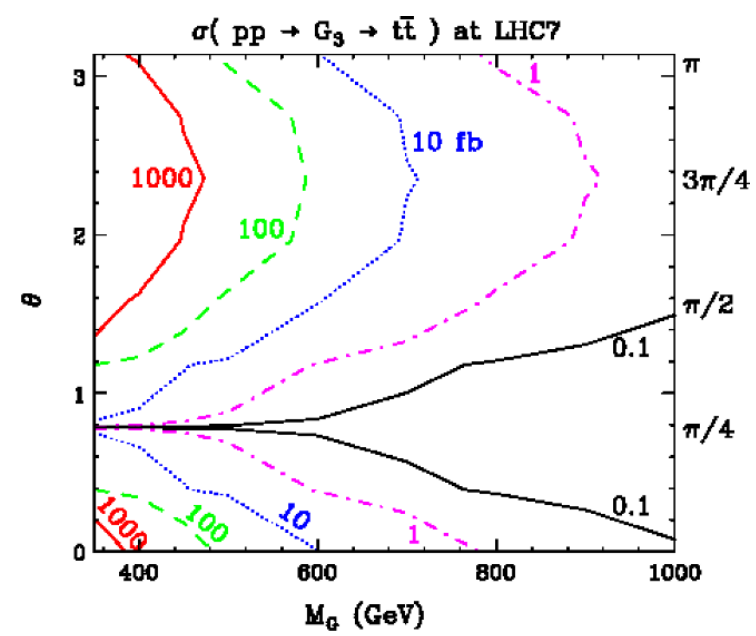

(a)

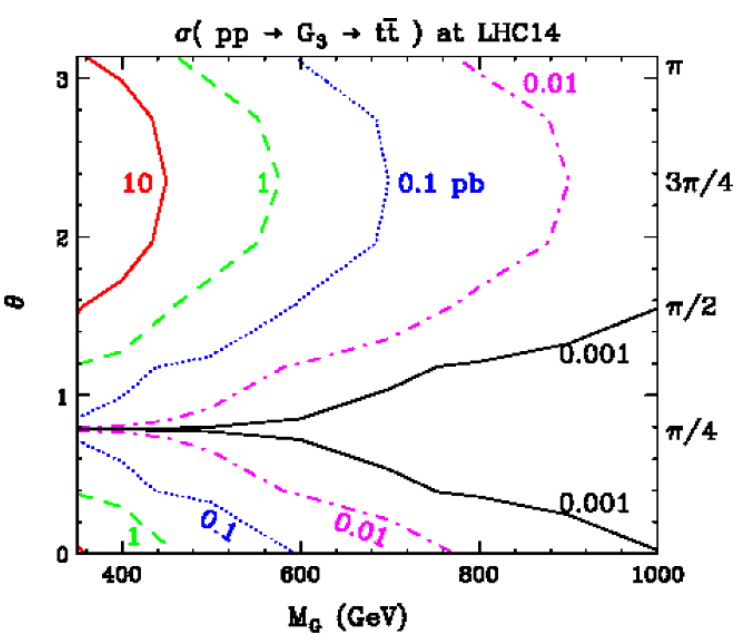

(b)

Figure 14. Lines of constant production cross section in the $M_{G^{-}} \theta$ plane using $c_{t}=1$ for the loop induced (Born) processes $p p \rightarrow G_{3} \rightarrow t \bar{t}$ at proton-proton colliders of $7 \mathrm{TeV}$ (14a) and $14 \mathrm{TeV}$ (14b) center-of-mass energy.

The bounds that apply to the $t \bar{t}+0 j$ calculations are more complicated to derive than in the previous case. As shown in eqs. (3.5) and (3.7), this is due to the fact that there are non-negligible contributions from the $t \bar{t}+1 j$ calculation that have to be taken into account once the extra jet is not or does not get resolved. Figure 14 shows the results for the LO contribution of the loop induced processes $p p \rightarrow G_{3} \rightarrow t \bar{t}$. To apply the corresponding cross section limits, which we have worked out in table 1 , we however need the approximate NLO cross section as explained in the previous section. ${ }^{6}$ These results are presented in figure 15, including the effect of the cross section bounds. Figure 15a shows the inclusive case at $7 \mathrm{TeV}$ which is described by eq. (3.5). The yellow shaded area denotes the region in parameter space that is excluded by the inclusive zero-jet measurement (I0) in table 1. Figure 15b shows the theoretical cross section predictions for $14 \mathrm{TeV}$. As before in figure 13, only in the $7 \mathrm{TeV}$ plot, the border of the excluded area can be associated with a line of constant cross section. This does not apply to the $14 \mathrm{TeV}$ plot.

Figures $15 \mathrm{c}$ and $15 \mathrm{~d}$ show the exclusive case, where the theoretical predictions are calculated according to eq. (3.7) and the yellow shaded area corresponds to the excluded area stemming from the exclusive zero-jet measurement (E0) in table 1. The direct comparison of figure 14 with figures $15 \mathrm{c}$ and $15 \mathrm{~d}$ reflects once more the important fact already demonstrated in figure 11b, namely that the contribution of the one-jet case, where the jet is not resolved, provides a substantial contribution to the zero-jet signal and cannot be neglected in a realistic study. This is the reason why the zero-jet cases provide constraints on the allowed parameter space that are stronger than those of the one-jet cases. Nevertheless, in both jet bin categories, the constraints on the parameter space are rather weak and a large

\footnotetext{
${ }^{6}$ The approximate NLO cross sections, i.e. the left-hand sides of eqs. (3.5) and (3.7), shown in figure 15 have been evaluated neglecting the $\mathcal{O}(10 \%)$ contribution from the $q g$ initial states in the calculation of the term $\sigma_{\text {Real }}^{\text {loose cuts }}$, which is described by the $t \bar{t}+1$ parton process at LO and the application of loose jet cuts.
} 


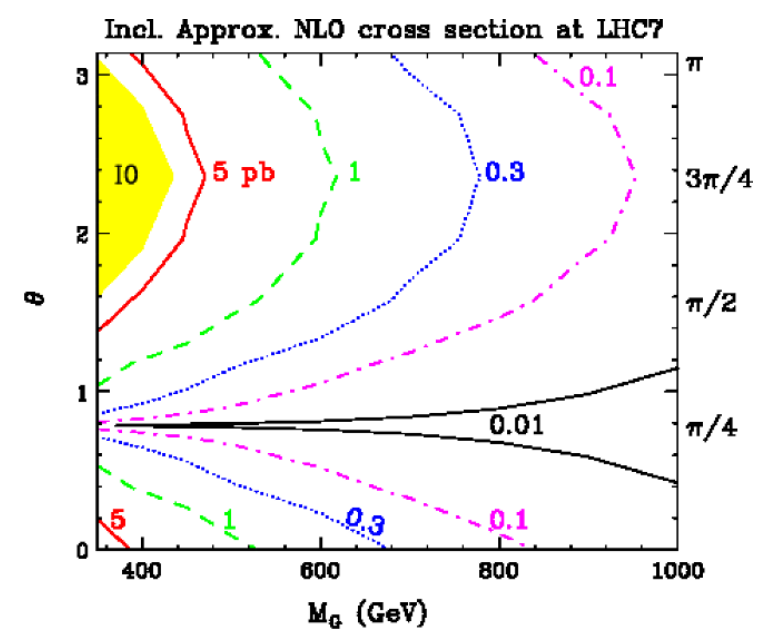

(a)

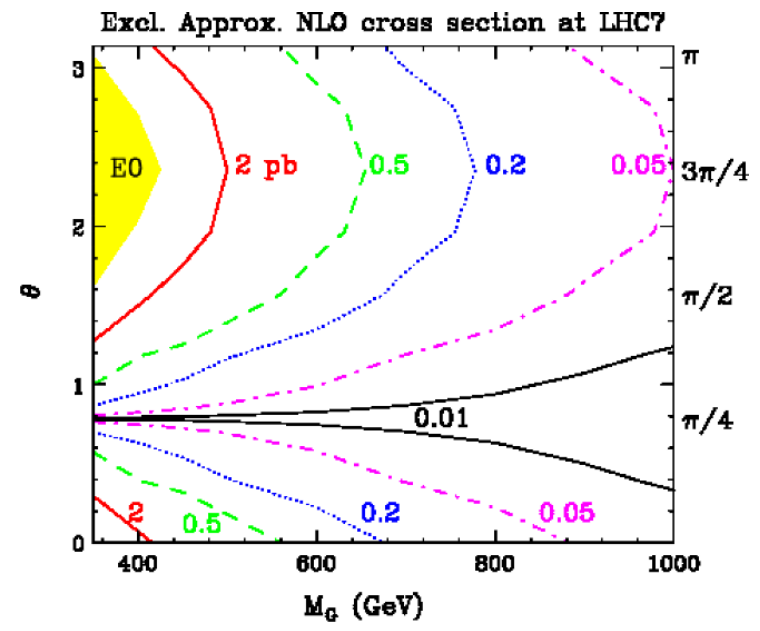

(c)
Incl. Approx. NLO cross gection at LHC14

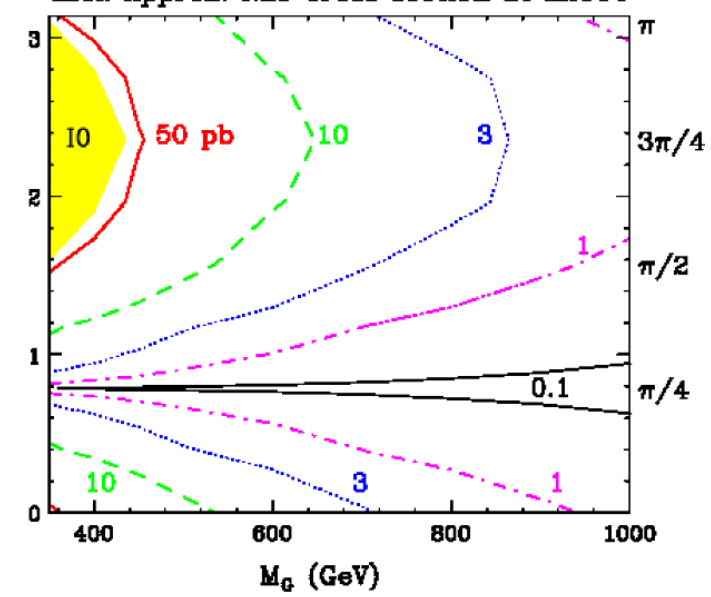

(b)

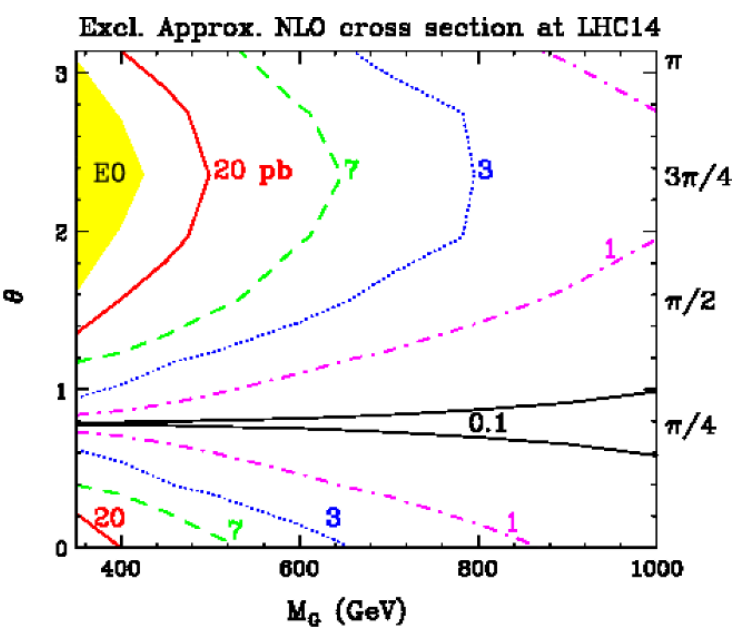

(d)

Figure 15. Approximate NLO cross sections in the loop induced mode for the inclusive (upper row) and exclusive (lower row) production of $G_{3} \rightarrow t \bar{t}$ using $c_{t}=1$ at a $7 \mathrm{TeV}$ (15a and 15c) and $14 \mathrm{TeV}$ (15b and 15d) proton-proton collider. Regions of parameter space excluded at the one-sigma level are indicated by the colored areas, and labelled using the jet categories of table 1 .

fraction of realistic scenarios cannot be excluded by present LHC data. We will need more precise limits at $8 \mathrm{TeV}$ as well as measurements at $14 \mathrm{TeV}$ in order to further constrain these types of models.

\subsubsection{LHC Run 2 prospects}

For the inclusive zero-jet case, the SM prediction for the top quark pair production cross section is known up to NNLO in QCD (including NNLL resummation effects) [47], whereas for the inclusive one-jet case, the cross section is computed at NLO [67-69]. Based on these predictions given in the literature, we can estimate the impact of the $G_{3}$ model on the $t \bar{t}+0,1$ jet final states. We should however stress that we cannot expect to obtain more than just a rough idea, since we build our arguments solely on a comparison of rather 
inclusive cross sections, i.e. no attempt is made to include information from differential observables/properties that prove to be different for the signal and the background. Comparing the SM predictions, $\sigma_{t \bar{t}}=953.6 \mathrm{pb}$ and $\sigma_{t \bar{t} j}=692 \mathrm{pb}$, with the results obtained in figures $15 \mathrm{~b}$ and $13 \mathrm{~b}$, we find rather low $S / B$ ratios of maximally $\sim 5 \%$ and $\sim 1.4 \%$ for the inclusive zero- and one-jet category, respectively. ${ }^{7}$ In the former case, the $S / B$ value corresponds to roughly the size of the systematic uncertainty of the theoretical calculation, while in the latter case it is considerably below the 6-9\% uncertainty of the theory prediction.

Instead of considering an analysis that requires at least one jet, we get a slightly better perspective by studying the situation in the exclusive zero-jet bin. We obtain a theoretical prediction for this contribution by subtracting the inclusive one-jet NLO result from the NNLO prediction for $\sigma_{t \bar{t}}$. Using simple error propagation, the uncertainty of this prediction amounts to $11-14 \%$. However, in a more sophisticated theoretical treatment, it can be expected to control the prediction at the $10 \%$ level or below. This implies that the minimal $S / B$ ratio has to be of similar size. The region in parameter space where this condition is fulfilled is represented by the purple shaded area in figure $16 \mathrm{~b}$. The same requirement applied to the total inclusive cross section, namely that $S / B \sim \Delta \sigma_{t \bar{t}} / \sigma_{t \bar{t}} \approx 0.05$, leads to weaker constraints on the parameters compared to the exclusive case, cf. figure 16a. The plots in figure 16 also show areas that correspond to a statistical significance $(S / \sqrt{B})$ larger than five, marking the discovery threshold. The areas shaded in green and yellow correspond to integrated luminosities of $10 \mathrm{fb}^{-1}$ and $100 \mathrm{fb}^{-1}$, respectively. We clearly see that in both the inclusive as well as exclusive case, most of the parameter plane is already covered after accumulating $10 \mathrm{fb}^{-1}$ in Run 2. This means that from a statistical point of view the LHC has the ability to discover a $G_{3}$ resonance in a wide region of parameter space, even with a low luminosity. We are not limited by a lack of statistics but by relatively large systematic uncertainties. Therefore, a refined strategy will have to exploit features in differential distributions where we might be able to overcome the systematic uncertainties.

\section{Conclusions}

Searches for various types of resonances at the LHC are very well motivated. These resonances may show up at an early stage of LHC Run 2 . However the null results at Run 1 indicate that their mass scale may be high or they may be hiding in an exotic corner of the phase space. The top quark is the heaviest particle in the Standard Model. It decays promptly to a $W$ boson and a $b$-quark before hadronization and may be vulnerable to new physics, which makes new physics searches in the top quark sector very appealing.

In this paper, we investigated a top-philic resonance, called $G_{3}$, which may be light. All existing analyses assume the production of a $t \bar{t}$ resonance from $q \bar{q}$ annihilation, which depends on its diquark coupling. We have proposed model-independent production channels both at the tree level and at the one-loop level. Our studies show that in spite of being loop suppressed, the one-loop channels dominate over the tree level processes and

\footnotetext{
${ }^{7}$ A somewhat better $S / B$ for the inclusive one-jet bin can be expected due to the differences in the jet parameters for the signal and background calculation.
} 


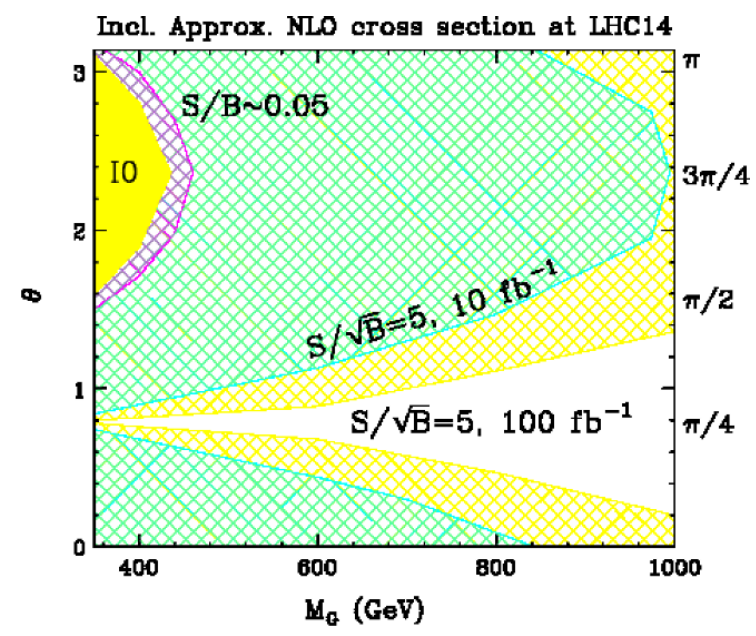

(a)

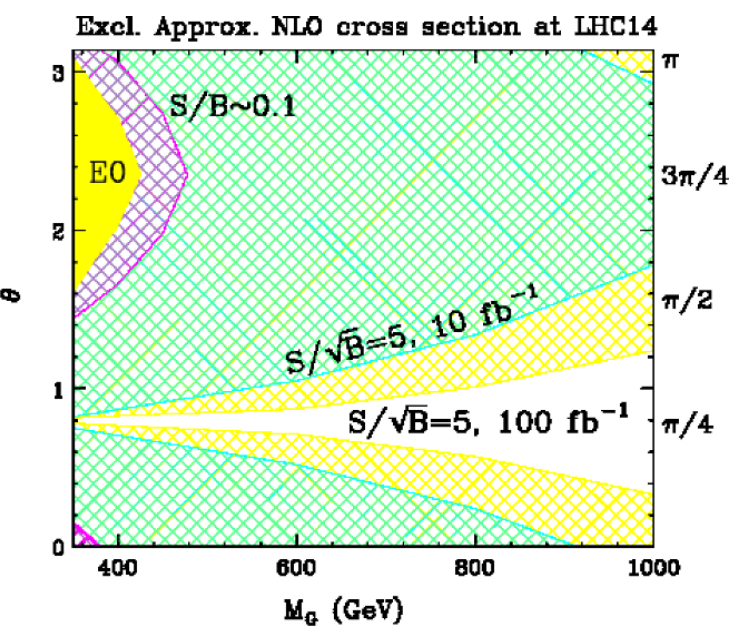

(b)

Figure 16. Possible reach of a search for $G_{3}$ at the $14 \mathrm{TeV}$ LHC in the loop induced mode for the inclusive (16a) and exclusive (16b) production of $G_{3} \rightarrow t \bar{t}$ using $c_{t}=1$. The shaded areas are used to indicate the one-sigma exclusions (in yellow) as in figure 15, the constraints stemming from $S / B$ considerations (in purple) and the parameter space that is accessible with a statistical significance of five-sigma and beyond using $10 \mathrm{fb}^{-1}$ (in green) and $100 \mathrm{fb}^{-1}$ (in light yellow) of LHC Run 2 data.

yield cross sections of up to several picobarns, strongly dependent on the chosen point in the model parameter space. While the tree level modes generate more exotic final states featuring multi-top quark signatures, the $G_{3}$ production via one loop leads to the appearance of individual top quark pairs, often accompanied by jets. In particular, the $t \bar{t}$ plus one-jet process is not suppressed compared to the zero-jet process as one would naively expect by counting orders in $\alpha_{\mathrm{s}}$. It rather supersedes the zero-jet case in its leading role and provides a non-negligible contribution to both measurements in the one-jet bin and in the zero-jet bin, depending on whether the additional jet is resolved or not. This is a strong hint for the presence of large NLO corrections that affect the $G_{3} \rightarrow t \bar{t}$ process and most likely lead to a significant enhancement of the signal cross section. However, as a full NLO treatment for this process is not feasible, we combined the $G_{3}$ and $G_{3} j$ calculations in a specific way to generate an approximate NLO prediction for the signal. The resulting jet cross sections should not exceed the size of experimental uncertainties on recent top quark pair measurements and hence were used to derive first constraints on the parameters of the model. While we obtain more stringent constraints from the inclusive zero-jet case, we yet find, regardless of the zero- or one-jet condition, that the majority of the parameter space has not been excluded by current LHC data. Certainly, stronger limits can be achieved by a reduction of the experimental uncertainties in $t \bar{t}$ measurements. More likely, they may be obtained from the exploitation of suitable differential distributions, although we want to emphasize that for this study, we restricted ourselves to setting limits according to the determination of total cross sections.

In our first attempt of obtaining estimates concerning the prospects for LHC Run 2, we utilized a simple $S / B$ and $S / \sqrt{B}$ statistics method considering ordinary top quark 
production in pairs and in association with jets as the only background sources for this analysis. Studying the most promising channels (i.e. the $G_{3} t \bar{t}$ process yielding four topquark final states and the loop induced $G_{3}$ production yielding inclusive $t \bar{t}$ final states), we identify the signal over background ratio, $S / B$, as the constraining quantity in our analysis whereas the statistical significance will be sufficiently large in all channels with just $10-100 \mathrm{fb}^{-1}$ of Run 2 data. Figures 12 and 16 provide us with a sketch of the $14 \mathrm{TeV}$ LHC sensitivity for each of these production channels. We find that the $t \bar{t}$ cross sections provide very stringent constraints on the axial coupling $(\theta=3 \pi / 4)$ whereas the multi-top quark production is particularly useful in testing the parameter space away from the axial coupling regime. This is because the $G_{3} t \bar{t}$ process does not have a chirality dependence. We also observe that the $G_{3} t \bar{t}$ process is in much better shape from an $S / B$ point of view than the loop level $t \bar{t}$ production, which suffers from larger backgrounds. However, the large cross sections of the loop induced modes leave more space to improve on $S / B$ by imposing suitable kinematical requirements. Moreover, the reconstruction of the $G_{3}$ resonance will be easier than for the more complicated final states of the multi-top quark production, and we should stress that an alleviated reconstruction procedure can be very useful in further background reduction. Taken the different strengths of the production channels, we find them to be complementary in both the $G_{3}$ discovery potential and the measurement of its properties. One should therefore take advantage of each channel and revisit them with full background analyses.

It should be stressed that the results of this paper follow from a detailed investigation of a color-singlet vector resonance possessing top-philic production and decay channels only. Its production cross section depends significantly on the chiral structure of the interaction. We note that at the one-loop level a color-singlet vector resonance can be produced on-shell if it is associated with a jet. In the corresponding $G_{3} \rightarrow t \bar{t}$ mode, it can however only be generated off-shell as a consequence of the Landau-Yang theorem. As shown in figure 9, the $G_{3}$ production cross section for a pure chiral interaction with a coupling strength of $\mathcal{O}(1)$ is comparable to that of a Standard-Model-like Higgs boson of similar mass, which represents an example of a color-singlet scalar particle (with the same coupling strength as the top quark Yukawa). As opposed to the vector case, the scalar production is affected neither by the Landau-Yang nor Furry's theorem, and we expect its phenomenology to be similar to that of a Standard Model Higgs boson. Unlike the color-singlet cases, a color-octet particle could have direct interactions with Standard Model gluons. This to some extent is model-dependent and thus beyond the scope of the current study. Nevertheless, in general we expect the production cross section of a color-octet particle (either scalar or vector) to be larger than that of a color-singlet. The main reason is that the color algebra associated with a color-octet leads to a larger color factor and an increased number of diagrams that can contribute to the production and decay of such a resonance.

Finally, although we have considered a $t \bar{t}$ resonance, which couples very weakly to the rest of the Standard Model, one could anticipate a sizable interaction of the resonance to $b \bar{b}$ as well. In this case, we end up with a richer spectrum of final states including more diverse signatures such as $b \bar{b} b \bar{b}, t \bar{t} b \bar{b}$, and $b \bar{b}+j$. Moreover, in the context of dark matter searches, where we assume that the $G_{3}$ couples to the dark matter in addition to its $t \bar{t}$ 
coupling, new channels open up producing new signatures such as $t \bar{t}+\mathbb{E}_{T}$ and $j+\mathbb{E}_{T}$. Including these new ideas, our first studies clearly show that a light top-philic resonance is capable of producing a rich phenomenology in final states at the LHC. We hence encourage the experimental collaborations to pursue a dedicated study on it.

\section{Acknowledgments}

NG wants to thank the other members of the GoSAm collaboration for their effort and for numerous valuable discussions. KK is supported in part by the US DOE Grant DE-FG0212ER41809 and by the University of Kansas General Research Fund allocation 2301566. JCP and SC are supported by the Basic Science Research Program through the National Research Foundation of Korea funded by the Ministry of Education, Science and Technology (based on contract numbers NRF-2013R1A1A2061561 and 2013R1A1A2064120, respectively).

Open Access. This article is distributed under the terms of the Creative Commons Attribution License (CC-BY 4.0), which permits any use, distribution and reproduction in any medium, provided the original author(s) and source are credited.

\section{References}

[1] U. Haisch and S. Westhoff, Massive Color-Octet Bosons: Bounds on Effects in Top-Quark Pair Production, JHEP 08 (2011) 088 [arXiv:1106.0529] [INSPIRE].

[2] B. Lillie, L. Randall and L.-T. Wang, The Bulk RS KK-gluon at the LHC, JHEP 09 (2007) 074 [hep-ph/0701166] [INSPIRE].

[3] B. Lillie, J. Shu and T.M.P. Tait, Kaluza-Klein Gluons as a Diagnostic of Warped Models, Phys. Rev. D 76 (2007) 115016 [arXiv:0706.3960] [INSPIRE].

[4] A.L. Fitzpatrick, J. Kaplan, L. Randall and L.-T. Wang, Searching for the Kaluza-Klein Graviton in Bulk RS Models, JHEP 09 (2007) 013 [hep-ph/0701150] [INSPIRE].

[5] K. Kong, S.C. Park and T.G. Rizzo, Collider Phenomenology with Split-UED, JHEP 04 (2010) 081 [arXiv: 1002.0602] [INSPIRE].

[6] T. Flacke, K. Kong and S.C. Park, Phenomenology of Universal Extra Dimensions with Bulk-Masses and Brane-Localized Terms, JHEP 05 (2013) 111 [arXiv:1303.0872] [INSPIRE].

[7] E.H. Simmons, Coloron phenomenology, Phys. Rev. D 55 (1997) 1678 [hep-ph/9608269] [INSPIRE].

[8] J. Bagger, C. Schmidt and S. King, Axigluon Production in Hadronic Collisions, Phys. Rev. D 37 (1988) 1188 [INSPIRE].

[9] ATLAS collaboration, A search for $t \bar{t}$ resonances in the lepton plus jets final state with ATLAS using $14 \mathrm{fb}^{-1}$ of pp collisions at $\sqrt{s}=8 \mathrm{TeV}$, ATLAS-CONF-2013-052 (2013) [INSPIRE].

[10] ATLAS collaboration, Search for $t \bar{t}$ resonances in the lepton plus jets final state with ATLAS using $4.7 \mathrm{fb}^{-1}$ of pp collisions at $\sqrt{s}=7 \mathrm{TeV}$, Phys. Rev. D 88 (2013) 012004 [arXiv: 1305.2756] [INSPIRE]. 


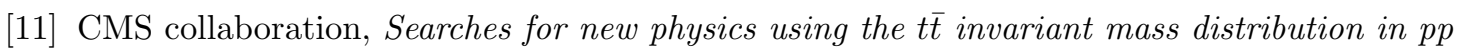
collisions at $\sqrt{s}=8$ TeV, Phys. Rev. Lett. 111 (2013) 211804 [arXiv:1309.2030] [INSPIRE].

[12] CMS collaboration, Search for $Z^{\prime}$ resonances decaying to $t \bar{t}$ in dilepton + jets final states in pp collisions at $\sqrt{s}=7$ TeV, Phys. Rev. D 87 (2013) 072002 [arXiv:1211.3338] [InSPIRE].

[13] New Physics Working Group, G. Brooijmans et al., New Physics at the LHC. A Les Houches Report: Physics at TeV Colliders 2009 - New Physics Working Group, CERN-PH-TH-2010-096 (2010) [arXiv:1005.1229] [INSPIRE].

[14] J. Alwall, M. Khader, A. Rajaraman, D. Whiteson and M. Yen, Searching for $Z^{\prime}$ bosons decaying to gluons, Phys. Rev. D 85 (2012) 115011 [arXiv:1202.4014] [INSPIRE].

[15] CDF collaboration, T. Aaltonen et al., Search for a heavy vector boson decaying to two gluons in $p \bar{p}$ collisions at $\sqrt{s}=1.96$ TeV, Phys. Rev. D 86 (2012) 112002 [arXiv:1210.5686] [INSPIRE].

[16] A. Belyaev, N.D. Christensen and A. Pukhov, CalcHEP 3.4 for collider physics within and beyond the Standard Model, Comput. Phys. Commun. 184 (2013) 1729 [arXiv:1207.6082] [INSPIRE].

[17] J. Alwall, M. Herquet, F. Maltoni, O. Mattelaer and T. Stelzer, MadGraph 5: Going Beyond, JHEP 06 (2011) 128 [arXiv:1106.0522] [INSPIRE].

[18] K. Kumar, T.M.P. Tait and R. Vega-Morales, Manifestations of Top Compositeness at Colliders, JHEP 05 (2009) 022 [arXiv:0901.3808] [INSPIRE].

[19] B. Lillie, J. Shu and T.M.P. Tait, Top Compositeness at the Tevatron and LHC, JHEP 04 (2008) 087 [arXiv:0712.3057] [INSPIRE].

[20] C. Han, N. Liu, L. Wu and J.M. Yang, Probing topcolor-assisted technicolor from top charge asymmetry and triple-top production at the LHC, Phys. Lett. B 714 (2012) 295 [arXiv:1203.2321] [INSPIRE].

[21] J.A. Aguilar-Saavedra and J. Santiago, Four tops and the $t \bar{t}$ forward-backward asymmetry, Phys. Rev. D 85 (2012) 034021 [arXiv:1112.3778] [InSPIRE].

[22] T. Plehn and T.M.P. Tait, Seeking Sgluons, J. Phys. G 36 (2009) 075001 [arXiv:0810.3919] [INSPIRE].

[23] B.A. Dobrescu, K. Kong and R. Mahbubani, Massive color-octet bosons and pairs of resonances at hadron colliders, Phys. Lett. B 670 (2008) 119 [arXiv:0709.2378] [INSPIRE].

[24] C.-R. Chen, W. Klemm, V. Rentala and K. Wang, Color Sextet Scalars at the CERN Large Hadron Collider, Phys. Rev. D 79 (2009) 054002 [arXiv:0811.2105] [InSPIRE].

[25] G. Cullen et al., GoSam-2.0: a tool for automated one-loop calculations within the Standard Model and beyond, Eur. Phys. J. C 74 (2014) 3001 [arXiv:1404.7096] [InSPIRE].

[26] G. Cullen et al., Automated One-Loop Calculations with GoSam, Eur. Phys. J. C 72 (2012) 1889 [arXiv: 1111.2034] [INSPIRE].

[27] P. Nogueira, Automatic Feynman graph generation, J. Comput. Phys. 105 (1993) 279 [INSPIRE].

[28] J.A.M. Vermaseren, New features of FORM, math-ph/0010025 [INSPIRE].

[29] G. Cullen, M. Koch-Janusz and T. Reiter, Spinney: A Form Library for Helicity Spinors, Comput. Phys. Commun. 182 (2011) 2368 [arXiv: 1008.0803] [INSPIRE]. 
[30] T. Reiter, Optimising Code Generation with haggies, Comput. Phys. Commun. 181 (2010) 1301 [arXiv:0907.3714] [INSPIRE].

[31] P. Mastrolia, E. Mirabella and T. Peraro, Integrand reduction of one-loop scattering amplitudes through Laurent series expansion, JHEP 06 (2012) 095 [Erratum ibid. 11 (2012) 128] [arXiv: 1203.0291] [INSPIRE].

[32] H. van Deurzen, G. Luisoni, P. Mastrolia, E. Mirabella, G. Ossola and T. Peraro, Multi-leg One-loop Massive Amplitudes from Integrand Reduction via Laurent Expansion, JHEP 03 (2014) 115 [arXiv:1312.6678] [INSPIRE].

[33] T. Peraro, Ninja: Automated Integrand Reduction via Laurent Expansion for One-Loop Amplitudes, Comput. Phys. Commun. 185 (2014) 2771 [arXiv:1403.1229] [InSPIRE].

[34] G. Ossola, C.G. Papadopoulos and R. Pittau, Reducing full one-loop amplitudes to scalar integrals at the integrand level, Nucl. Phys. B 763 (2007) 147 [hep-ph/0609007] [INSPIRE].

[35] P. Mastrolia, G. Ossola, C.G. Papadopoulos and R. Pittau, Optimizing the Reduction of One-Loop Amplitudes, JHEP 06 (2008) 030 [arXiv:0803.3964] [INSPIRE].

[36] G. Ossola, C.G. Papadopoulos and R. Pittau, On the Rational Terms of the one-loop amplitudes, JHEP 05 (2008) 004 [arXiv:0802.1876] [INSPIRE].

[37] P. Mastrolia, G. Ossola, T. Reiter and F. Tramontano, Scattering Amplitudes from Unitarity-based Reduction Algorithm at the Integrand-level, JHEP 08 (2010) 080 [arXiv: 1006.0710] [INSPIRE].

[38] G. Heinrich, G. Ossola, T. Reiter and F. Tramontano, Tensorial Reconstruction at the Integrand Level, JHEP 10 (2010) 105 [arXiv: 1008.2441] [INSPIRE].

[39] T. Binoth, J.-P. Guillet, G. Heinrich, E. Pilon and T. Reiter, Golem95: A Numerical program to calculate one-loop tensor integrals with up to six external legs, Comput. Phys. Commun. 180 (2009) 2317 [arXiv:0810.0992] [INSPIRE].

[40] G. Cullen et al., Golem95C: A library for one-loop integrals with complex masses, Comput. Phys. Commun. 182 (2011) 2276 [arXiv:1101.5595] [INSPIRE].

[41] J.P. Guillet, G. Heinrich and J.F. von Soden-Fraunhofen, Tools for NLO automation: extension of the golem95C integral library, Comput. Phys. Commun. 185 (2014) 1828 [arXiv: 1312.3887] [INSPIRE].

[42] A. van Hameren, OneLOop: For the evaluation of one-loop scalar functions, Comput. Phys. Commun. 182 (2011) 2427 [arXiv:1007.4716] [INSPIRE].

[43] F. Maltoni and T. Stelzer, MadEvent: Automatic event generation with MadGraph, JHEP 02 (2003) 027 [hep-ph/0208156] [INSPIRE].

[44] CMS collaboration, Search for standard model production of four top quarks in the lepton + jets channel in pp collisions at $\sqrt{s}=8 \mathrm{TeV}$, JHEP 11 (2014) 154 [arXiv:1409.7339] [INSPIRE].

[45] ATLAS collaboration, Search for exotic same-sign dilepton signatures ( $b^{\prime}$ quark, $T_{5 / 3}$ and four top quarks production) in $4.7 / \mathrm{fb}$ of pp collisions at $\sqrt{s}=7 \mathrm{TeV}$ with the ATLAS detector, ATLAS-CONF-2012-130 (2012) [INSPIRE].

[46] G. Bevilacqua and M. Worek, Constraining BSM Physics at the LHC: Four top final states with NLO accuracy in perturbative QCD, JHEP 07 (2012) 111 [arXiv:1206.3064] [INSPIRE]. 
[47] M. Czakon, P. Fiedler and A. Mitov, Total Top-Quark Pair-Production Cross Section at Hadron Colliders Through $O\left(\alpha_{S}^{4}\right)$, Phys. Rev. Lett. 110 (2013) 252004 [arXiv:1303.6254] [INSPIRE].

[48] CDF and D0 collaborations, T.A. Aaltonen et al., Combination of measurements of the top-quark pair production cross section from the Tevatron Collider, Phys. Rev. D 89 (2014) 072001 [arXiv: 1309.7570] [INSPIRE].

[49] CDF collaboration, T. Schwarz, R. Erbacher, J. Huston, M. Hussein and S. Mrenna, Measurement of the $t \bar{t}+$ jet Cross section with $4.1 \mathrm{fb}^{-1}$, CDF Note 9850 (2009).

[50] ATLAS collaboration, Measurement of the top quark pair production cross-section with ATLAS in pp collisions at $\sqrt{s}=7 \mathrm{TeV}$ in the single-lepton channel using semileptonic $b$ decays, ATLAS-CONF-2012-131 (2012) [INSPIRE].

[51] CMS collaboration, Measurement of the t $\bar{t}$ production cross section in the dilepton channel in pp collisions at $\sqrt{s}=7 \mathrm{TeV}$, JHEP 11 (2012) 067 [arXiv:1208.2671] [INSPIRE].

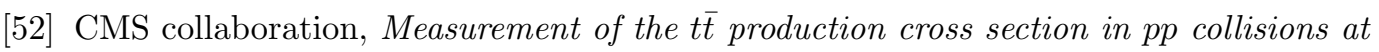
$\sqrt{s}=7 \mathrm{TeV}$ with lepton + jets final states, Phys. Lett. B 720 (2013) 83 [arXiv:1212.6682] [INSPIRE].

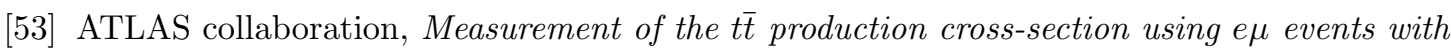
b-tagged jets in pp collisions at $\sqrt{s}=7$ and 8 TeV with the ATLAS detector, Eur. Phys. J. C 74 (2014) 3109 [arXiv:1406.5375] [INSPIRE].

[54] ATLAS collaboration, Simultaneous measurements of the $t \bar{t}, W^{+} W^{-}$and $Z / \gamma^{*} \rightarrow \tau \tau$ production cross-sections in pp collisions at $\sqrt{s}=7 \mathrm{TeV}$ with the ATLAS detector, CERN-PH-EP-2014-119 (2014) [INSPIRE].

[55] ATLAS collaboration, Measurement of the top quark pair production cross section in the single-lepton channel with ATLAS in proton-proton collisions at $8 \mathrm{TeV}$ using kinematic fits with b-tagging, ATLAS-CONF-2012-149 (2012) [INSPIRE].

[56] CMS Collaboration, Top pair cross section in e/mu + jets at 8 TeV, CMS-PAS-TOP-12-006 (2012) [INSPIRE].

[57] ATLAS collaboration, Combination of ATLAS and CMS top-quark pair cross section measurements using up to $1.1 \mathrm{fb}^{-1}$ of data at $7 \mathrm{TeV}$, ATLAS-CONF-2012-134 (2012) [INSPIRE].

[58] ATLAS collaboration, Combination of ATLAS and CMS top quark pair cross section measurements in the e $\mu$ final state using proton-proton collisions at $\sqrt{s}=8 \mathrm{TeV}$, ATLAS-CONF-2014-054 (2014) [INSPIRE].

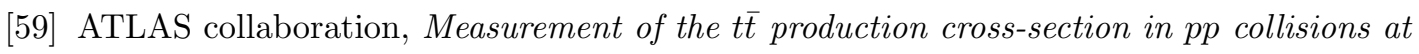
$\sqrt{s}=8 \mathrm{TeV}$ using e $\mu$ events with b-tagged jets, ATLAS-CONF-2013-097 (2013) [INSPIRE].

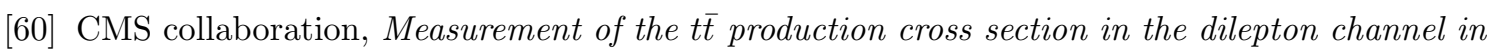
pp collisions at $\sqrt{s}=8 \mathrm{TeV}$, JHEP 02 (2014) 024 [Erratum ibid. 02 (2014) 102] [arXiv: 1312.7582] [INSPIRE].

[61] M. Czakon, M.L. Mangano, A. Mitov and J. Rojo, Constraints on the gluon PDF from top quark pair production at hadron colliders, JHEP 07 (2013) 167 [arXiv:1303.7215] [INSPIRE].

[62] ATLAS collaboration, Measurement of the cross section in $t \bar{t}+$ jets using kinematics fit method with the ATLAS detector, ATLAS-CONF-2012-083 (2012) [INSPIRE]. 
[63] CMS collaboration, Measurement of jet multiplicity distributions in $t \bar{t}$ production in $p p$ collisions at $\sqrt{s}=7 \mathrm{TeV}$, Eur. Phys. J. C 74 (2014) 3014 [arXiv:1404.3171] [InSPIRE].

[64] ATLAS collaboration, Measurement of the t $\bar{t}$ production cross-section as a function of jet multiplicity and jet transverse momentum in $7 \mathrm{TeV}$ proton-proton collisions with the ATLAS detector, JHEP 01 (2015) 020 [arXiv: 1407.0891] [INSPIRE].

[65] ATLAS collaboration, Measurement of the jet multiplicity in top anti-top final states produced in $7 \mathrm{TeV}$ proton-proton collisions with the ATLAS detector, ATLAS-CONF-2012-155 (2012) [INSPIRE].

[66] CMS Collaboration, Measurement of the Jet Multiplicity in dileptonic Top Quark Pair Events at $8 \mathrm{TeV}$, CMS-PAS-TOP-12-041 (2013) [INSPIRE].

[67] S. Dittmaier, P. Uwer and S. Weinzierl, NLO QCD corrections to $t \bar{t}+$ jet production at hadron colliders, Phys. Rev. Lett. 98 (2007) 262002 [hep-ph/0703120] [INSPIRE].

[68] S. Dittmaier, P. Uwer and S. Weinzierl, Hadronic top-quark pair production in association with a hard jet at next-to-leading order QCD: Phenomenological studies for the Tevatron and the LHC, Eur. Phys. J. C 59 (2009) 625 [arXiv:0810.0452] [inSPIRE].

[69] S. Alioli, S.-O. Moch and P. Uwer, Hadronic top-quark pair-production with one jet and parton showering, JHEP 01 (2012) 137 [arXiv:1110.5251] [INSPIRE]. 